\title{
Theoretical Estimation of the Superrotation Strength in an Idealized Quasi-Axisymmetric Model of Planetary Atmospheres
}

\author{
Hiroki YAMAMOTO and Shigeo YODEN \\ Graduate School of Science, Kyoto University, Kyoto, Japan \\ (Manuscript received 12 April 2012, in final form 14 November 2012)
}

\begin{abstract}
This paper presents a theoretical estimation of the strength of equatorial superrotation in planetary atmospheres by exploring a quasi-axisymmetric system that is zonally averaged primitive equations for a dry Boussinesq fluid on a rotating hemisphere with the effects of nonaxisymmetric eddies parameterized by eddy diffusion. The fluid is forced by Newtonian heating and cooling, and the horizontal eddy diffusion of momentum is assumed to be much stronger than the vertical one. In this system, the superrotation is maintained by the Gierasch mechanism, which possibly explains the superrotation of the Venus atmosphere by angular momentum transport due to the mean meridional circulation and horizontal diffusion. For the estimation, a quintic equation for a scalar measure of the superrotation strength is developed from the primitive equations. The quintic equation estimates the superrotation strength by its unique positive solution, which depends only on three nondimensional parameters: the external thermal Rossby number, the ratio of the radiative relaxation time to the timescale for the vertical diffusion, and the ratio of the planetary rotation period to the geometric mean of the timescales for the horizontal and vertical diffusion. The parameter dependence of the dominant dynamical balance is also investigated. The balance is a cyclostrophic, geostrophic, or horizontal diffusion balance, and in each balance, the equator-to-pole temperature difference is either nearly equal to that in the radiative-convective equilibrium state or significantly reduced by thermal advection.

Steady-state or statistically steady-state solutions of the primitive equations are obtained by numerical timeintegrations for a wide parameter range covering many orders of magnitude. The numerical solutions show that the theoretical estimates have a relative error of less than $50 \%$, which is very small compared with the superrotation strength varying five orders depending on the external parameters, and show that the estimation is valid.
\end{abstract}

Keywords superrotation; general circulation; planetary atmospheres

\section{Introduction}

The Venus atmosphere is rotating much faster than its planetary rotation. Titan, Saturn's largest moon, has a similar fast-rotating atmosphere. The mechanism of such strong superrotation is a mystery of the general circulation of planetary atmospheres. The observed equatorial westward wind of the Venus atmosphere achieves a speed of $\sim 100 \mathrm{~m} \mathrm{~s}^{-1}$ at the cloud-top level

Corresponding author: Hiroki Yamamoto, Institute of Space and Astronautical Science, Japan Aerospace Exploration Agency (current affiliation), 3-1-1, Yoshinodai, Chuou, Sagamihara, Kanagawa 252-5210, Japan.

E-mail: hiroki@gfd-dennou.org

(C)2013, Meteorological Society of Japan of $\sim 65 \mathrm{~km}$ altitude (e.g., Counselman III et al. 1980). This zonal wind is about 55 times faster than the planetary rotation speed at the equator. Similarly, in Titan's atmosphere, equatorial eastward wind achieves a speed of $\sim 100 \mathrm{~m} \mathrm{~s}^{-1}$ at $\sim 120 \mathrm{~km}$ altitude (Bird et al. 2005); this is 8.3 times faster than the planetary rotation speed. Because the surface friction and vertical diffusion decelerate a superrotating atmosphere, some mechanism supplying angular momentum to the atmosphere must exist to maintain the superrotation.

The maintenance mechanism of the superrotation remains unclear mainly because observations of planetary atmospheres are limited. Many researchers in the field of planetary atmospheres hoped that the 
Japanese Venus Climate Orbiter (Akatsuki), which was expected to become the first extraterrestrial weather satellite (Nakamura et al. 2007, 2011), would provide observational data at high resolution in space and time. However, Akatsuki failed the first trial to enter the orbit around Venus on December 7, 2010.

To compensate for the lack of observational data, GCMs for realistic atmospheres of Venus and Titan have been developed. Young and Pollack (1977) first attempted to simulate the Venus superrotation with a GCM. Subsequently, many GCMs have succeeded in obtaining superrotational states (Yamamoto and Takahashi 2003; Hollingsworth et al. 2007; Lee et al. 2007), but these GCMs have been forced with simplified and unrealistically strong radiative heating. Lebonnois et al. (2010) have recently developed a GCM with a sophisticated radiative transfer code, but their simulated zonal mean zonal wind under the cloud layer is much slower than observed. Even if GCMs succeed in simulating the superrotation, we need to interpret their numerical results dynamically in order to understand the maintenance mechanism of the superrotation.

Construction of a theoretical model that grasps the essence of the superrotation mechanism is indispensable to understand the general circulation of planetary atmospheres, which are highly nonlinear systems. For the superrotation mechanism, several hypotheses have been proposed (for a summary, see Gierasch et al. 1997). Among the hypotheses, two convincing ones are Gierasch's (1975) hypothesis, which we briefly review below, and the thermal tide hypothesis proposed by Fels and Lindzen (1974).

Gierasch (1975) presented a possible mechanism of the superrotation by considering a quasi-axisymmetric model. Here, "quasi-axisymmetric model" means a system describing the zonally averaged component of a 3D flow with a parameterization of the nonaxisymmetric eddy mixing by eddy diffusion (Read 1986b). ${ }^{1}$ Gierasch proposed that angular momentum is pumped vertically by the mean meridional circulation and is transported toward the equator by horizontal eddy diffusion. It is required for Gierasch's hypothesis that the relaxation time for horizontal diffusion must be much shorter than both the turnover time of the

\footnotetext{
${ }^{1}$ Strictly speaking, Read (1986b) used the term "quasiaxisymmetric model" for a zonally averaged model using the Newtonian formulation of diffusion for eddy diffusion; however, we use the term not only for such a model using the Newtonian formulation but also for that using other formulations.
}

meridional circulation and the relaxation time for vertical diffusion. It has been shown that in a nonaxisymmetric atmosphere with slow planetary rotation, equatorward transport of angular momentum can be caused by an equatorial eddy source, such as barotropic instability (Rossow and Willams 1979; Iga and Matsuda 1999; Luz and Hourdin 2003) and other horizontal shear instability with destabilized Kelvin modes (Iga and Matsuda 2005). Many studies with various GCMs have been conducted and have supported Gierasch's hypothesis (e.g., Hourdin et al. 1995; Del Genio and Zhou 1996; Yamamoto and Takahashi 2003).

Matsuda $(1980,1982)$ theoretically and numerically studied Gierasch's hypothesis by using a two-layer highly truncated spectral model with a few fundamental modes in horizontal. He explored the dependence of superrotation strength, defined as the ratio of the mean zonal wind to the planetary rotation speed at the equator, on horizontal and vertical eddy diffusion coefficients of momentum, the planetary rotation rate, and a specified equator-to-pole temperature difference. In addition, with his idealized simple model, he for the first time demonstrated the possibility of multiple equilibrium states in the general circulation of the Venus atmosphere. Use of a hierarchy of numerical models, not only complex GCMs but also idealized simple models, is important for the balanced approach that is necessary to understand the mechanism of complex and highly nonlinear phenomena, such as general circulation of planetary atmospheres (e.g., Hoskins 1983; Held 2005).

In the present study, we theoretically and numerically explore an idealized quasi-axisymmetric system to investigate the parameter dependence of superrotation strength without the following assumption used in both Gierasch (1975) and Matsuda (1980, 1982). They assumed a specified equator-to-pole temperature difference, even though the temperature difference can be decreased by meridional thermal advection. In this study, on the other hand, we drop the assumption and treat the equator-to-pole temperature difference as an internal variable. The idealized system used in this study is quasi-axisymmetric primitive equations for a Boussinesq fluid forced by Newtonian heating and cooling. This is basically the same system as that used by Matsuda $(1980,1982)$ after severe truncation with retaining a few modes. In this system, the superrotation can be maintained by the angular momentum pumped vertically by the mean meridional circulation and transported toward the equator by strong horizontal eddy diffusion. Hereafter, we use the term "Gierasch 
mechanism" to refer to this maintenance mechanism without fixing the equator-to-pole temperature difference.

Because the primitive equations are nonlinear PDEs, in general, we cannot predict the parameter dependence of their solutions analytically. A usual way to reveal the dependence is to obtain approximate numerical solutions of the PDEs over a wide range of external parameters. In this study, however, we attempt to predict the parameter dependence of superrotation strength with a theoretical model that consists of four relationship equations for the following four scales: the zonal wind at the top, the meridional winds at the top and just above the surface, and the equator-to-pole difference of the vertically averaged potential temperature. The four equations constitute simultaneous equations with the four scales as scalar unknowns. Their solutions give the theoretical estimates of the four scales. Then, the estimates are compared with numerical results obtained by timeintegrations of a discretized set of the original primitive equations. The parameter dependence of the dominant dynamical balance is also investigated.

Our theoretical model potentially captures the dynamical essence of the superrotation, although our quantitative results might not be applied directly to real planetary atmospheres because the system analyzed in this study is a highly idealized one. We believe that our idealized model will play a unique role in the balanced approach to understand the dynamics of the superrotation.

In the following section, we provide a description of the governing equations of the idealized system. The theoretical model is presented and analyzed in Section 3. In Section 4, the theoretical model is compared with the numerical solutions of the primitive equations. Discussion and summary are presented in Sections 5 and 6 , respectively.

\section{Governing equations of the system}

We consider the quasi-axisymmetric form of primitive equations for a dry Boussinesq fluid on a rotating hemisphere. This system describes the zonally averaged component of a 3D flow with a parameterization of nonaxisymmetric eddy mixing by eddy diffusion. The eddy diffusion terms of momentum and heat are formulated in analogy with molecular diffusion. However, following Gierasch (1975), we assume that horizontal diffusion efficiently transports momentum to homogenize angular velocity (i.e., down-gradient with respect to angular velocity) but does not transport heat. The fluid is forced by
Newtonian heating and cooling, that is, a simple form for radiative heating and cooling proportional to the difference between the fluid temperature and a prescribed radiative-convective equilibrium temperature. The governing equations are written as follows:

$$
\begin{aligned}
& \frac{\partial u}{\partial t}+\frac{v}{a} \frac{\partial u}{\partial \phi}+w \frac{\partial u}{\partial z}-\frac{u v \tan \phi}{a}-2 \Omega v \sin \phi \\
& =\nu_{H} D_{H}(u)+\nu_{V} \frac{\partial^{2} u}{\partial z^{2}}, \\
& \frac{\partial v}{\partial t}+\frac{v}{a} \frac{\partial v}{\partial \phi}+w \frac{\partial v}{\partial z}+\frac{u^{2} \tan \phi}{a}+2 \Omega u \sin \phi \\
& =-\frac{1}{a} \frac{\partial \Phi}{\partial \phi}+\nu_{H} D_{H}(v)+\nu_{V} \frac{\partial^{2} v}{\partial z^{2}}, \\
& \frac{\partial \Phi}{\partial z}=g \frac{\theta-\Theta_{0}}{\Theta_{0}}, \\
& \frac{\partial \theta}{\partial t}+\frac{v}{a} \frac{\partial \theta}{\partial \phi}+w \frac{\partial \theta}{\partial z}=-\frac{\theta-\theta_{e}}{\tau}+\kappa_{V} \frac{\partial^{2} \theta}{\partial z^{2}}, \\
& \frac{1}{a \cos \phi} \frac{\partial}{\partial \phi}(v \cos \phi)+\frac{\partial w}{\partial z}=0,
\end{aligned}
$$

where

$$
\begin{aligned}
D_{H}(u) \equiv & \frac{1}{a^{2} \cos \phi} \frac{\partial}{\partial \phi}\left(\cos \phi \frac{\partial u}{\partial \phi}\right)-\frac{u}{a^{2} \cos ^{2} \phi}+\frac{2 u}{a^{2}}, \\
D_{H}(v) \equiv & \frac{1}{a^{2} \cos \phi} \frac{\partial}{\partial \phi}\left(\cos \phi \frac{\partial v}{\partial \phi}\right)-\frac{v}{a^{2} \cos ^{2} \phi} \\
& +\frac{1}{a} \frac{\partial}{\partial \phi}\left[\frac{1}{a \cos \phi} \frac{\partial}{\partial \phi}(v \cos \phi)\right]+\frac{2 v}{a^{2}} .
\end{aligned}
$$

The variables $u, v$, and $w$ are the zonal, meridional, and vertical components of the velocity, respectively, $\theta$ is the potential temperature, and $\Phi \equiv p^{\prime} / \rho_{0}$, where $p^{\prime}$ is the dynamic pressure and $\rho_{0}$ is the constant density. The independent variables $\phi, z$, and $t$ are the latitude, height, and time, respectively. The constant $a$ is the planetary radius, $\Omega$ is the angular velocity of the planetary rotation, $g$ is the gravitational acceleration, $\nu_{H}$ and $\nu_{V}$ are the horizontal and vertical diffusion coefficients of momentum, respectively, $\kappa_{V}$ is the vertical thermal diffusion coefficient, $\tau$ is the time constant for Newtonian heating and cooling, and $\Theta_{0}$ is a reference potential temperature. The values of $\nu_{H}, \nu_{V}$, $\kappa_{V}$, and $\tau$ are assumed to be constant for simplicity. The horizontal diffusion terms, $D_{H}(u)$ and $D_{H}(v)$, are defined in the form to conserve angular momentum (Becker 2001) and are equivalent to those used in Gierasch (1975). The radiative-convective equilibrium potential 
temperature $\theta_{e}$ for Newtonian heating and cooling is given on the basis of the second Legendre polynomial $P_{2}(\sin \phi)=\left(3 \sin ^{2} \phi-1\right) / 2$ as

$$
\theta_{e} \equiv \Theta_{0}\left[1-\Delta_{H}\left(\sin ^{2} \phi-\frac{1}{3}\right)\right] \text {, }
$$

where $\Delta_{H}$ is the fractional change in potential temperature from the equator to the pole, and the global mean of $\theta_{e}$ is $\Theta_{0}$.

The vertical boundary conditions are stress-free at the top boundary $(z=H)$, no-slip at the bottom boundary $(z=0)$, and no vertical mass or heat flux at both top and bottom boundaries:

$$
\frac{\partial u}{\partial z}=\frac{\partial v}{\partial z}=w=\frac{\partial \theta}{\partial z}=0 \quad \text { at } \quad z=H(\text { top }),
$$

and

$$
u=v=w=\frac{\partial \theta}{\partial z}=0 \quad \text { at } \quad z=0 \text { (bottom). }
$$

The lateral boundary conditions are no horizontal mass, momentum, or heat flux at both the equator $(\phi=$ 0 ) and the pole $(\phi=\pi / 2)$ under the assumption of equatorial symmetry:

$$
v=\frac{\partial u}{\partial \phi}=\frac{\partial w}{\partial \phi}=\frac{\partial \theta}{\partial \phi}=0 \quad \text { at } \quad \phi=0, \frac{\pi}{2} .
$$

We should mention that the formulation of the eddy diffusion terms is a key assumption in this study. The horizontal diffusion term (6) in the zonal momentum equation acts in the direction of down-gradient with respect to angular velocity; that is, it can transport absolute angular momentum per unit mass defined by $M(\phi, z) \equiv[u(\phi, z)+a \Omega \cos \phi] a \cos \phi$ in its up-gradient direction. This diffusion term plays an important role in generating and maintaining the superrotation. We will discuss the formulation of the eddy diffusion terms in Section 5.1.

The horizontal diffusion term that can transport $M$ in its up-gradient direction (mostly equatorward) seems to be valid in cases that planetary rotation is slow, as mentioned in the previous section. On the other hand, in fast planetary rotation cases, baroclinic instability in mid-latitudes becomes the dominant eddy source, which causes angular momentum transport toward mid-latitudes. Therefore, in such cases, the formulation of the eddy diffusion that transports $M$ equatorward is not realistic. In this study, however, we use the same formulation in both slow and fast planetary rotation cases for a simple parameter study focusing on parameter dependence of the system described in this section. A discussion on the fast rotation cases is presented in Section 5.5.

\section{Theoretical model}

In this section, we develop a theoretical model that consists of four relationship equations, obtained from Eqs. (1)-(11), for the following four nondimensional scalar variables that characterize the general circulation of planetary atmospheres:

$$
\begin{aligned}
S & \equiv \frac{U}{a \Omega}, \quad R_{v B} \equiv \frac{V_{B}}{a \Omega}, \quad R_{v T} \equiv \frac{V_{T}}{a \Omega}, \quad \text { and } \\
\beta & \equiv \frac{\Delta \Theta}{\Theta_{0} \Delta_{H}} .
\end{aligned}
$$

Here, $S$ represents the superrotation strength with $U$ as a scale for the zonal wind at the top, $R_{v B}$ and $R_{v T}$ represent the Rossby numbers scaled with $V_{B}$ and $V_{T}$ as scales for the meridional winds just above the surface and at the top, respectively, and $\beta$ represents the ratio of the equator-to-pole difference of vertically averaged potential temperature $\Delta \Theta$ to that in the radiativeconvective equilibrium state $\Theta_{0} \Delta_{H}$. Note that we use the notation $\beta$ following Yamamoto et al. (2009); $\beta$ does not represent the meridional gradient of the Coriolis parameter in the present study. We regard the four relationship equations as a set of algebraic equations whose solutions give theoretical estimates. The equations are developed in the next subsection, which is followed by a subsection on analysis with a further simplification of the algebraic equations.

\subsection{Development of a set of algebraic equations}

To develop a set of algebraic equations, we simplify three of the governing equations under a steady-state assumption in the following order: the zonal momentum equation (1), the thermodynamic equation (4), and the meridional momentum equation (2).

\section{a. Zonal momentum equation}

We simplify Eq. (1) for a steady state $(\partial / \partial t=0)$ to obtain two equations; one describes a relationship between $S$ and $R_{v B}$, and the other describes that between $S$ and $R_{v T}$. With absolute angular momentum per unit mass $M(\phi, z)$, the meridional integral of Eq. (1) from the equator $(\phi=0)$ to the pole $(\phi=\pi / 2)$ becomes

$$
\int_{0}^{\pi / 2} \frac{\partial}{\partial z}(w M) \cos \phi d \phi=\nu_{V} \int_{0}^{\pi / 2} \frac{\partial^{2} M}{\partial z^{2}} \cos \phi d \phi .
$$

Here, the integral of the meridional advection term vanishes because of the lateral boundary conditions (11), and the integral of the horizontal diffusion term vanishes because it just redistributes $M$ meridionally. Integrating Eq. (13) vertically from $z=0$ to $z=H$, we 
obtain

$$
\left.\int_{0}^{\pi / 2} \frac{\partial M}{\partial z}\right|_{z=0} \cos \phi d \phi=0,
$$

by using the vertical boundary conditions (9) and (10). Equation (14) implies that the globally averaged surface flux of angular momentum becomes zero under the steady-state condition.

Now, we let $M_{0}(z) \equiv \int_{0}^{\pi / 2} M_{r}(\phi, z) \cos \phi d \phi$, where $M_{r}$ $(\phi, z) \equiv u(\phi, z) a \cos \phi$ is the relative angular momentum. From Eq. (14) and the vertical boundary conditions, $\partial u /\left.\partial z\right|_{z=H}=u(\phi, 0)=0$, we obtain the boundary conditions with respect to $M_{0}(z)$ as follows:

$$
\left.\frac{\partial M_{0}}{\partial z}\right|_{z=0}=\left.\frac{\partial M_{0}}{\partial z}\right|_{z=H}=M_{0}(0)=0 .
$$

By considering Eq. (15), we assume that $M_{0}$ is written as $M_{0}(z)=U a[1-\cos (\pi z / H)] / 2$, where $U$ is a scale for $u$ at the top $(z=H)$. The assumed form is the first mode of $M_{0}$ expanded with cosine series as $M_{0}(z)=\sum_{m=0}^{\infty} b_{m}$ $\{1-\cos [(2 m+1) \pi z / H]\}$, where $b_{m}$ is an expansion coefficient.

Let us consider Eq. (13) just above the surface, $z=h$ $\approx 0$, where the zonal and vertical components of the velocity almost vanish (i.e., $M_{r} \approx 0$ and $w \approx 0$ at $z=h$ ). The LHS of Eq. (13) at $z=h$ becomes

$$
\begin{aligned}
& \text { [LHS of Eq. (13) at } z=h] \\
& \qquad\left.\int_{0}^{\pi / 2}\left(M \frac{\partial w}{\partial z}+w \frac{\partial M}{\partial z}\right)\right|_{z=h} \cos \phi d \phi \\
& \left.\quad \approx a^{2} \Omega \int_{0}^{\pi / 2} \frac{\partial w}{\partial z}\right|_{z=h} \cos ^{3} \phi d \phi \\
& \quad=-a \Omega \int_{0}^{\pi / 2} \frac{\partial}{\partial \phi}[v(\phi, h) \cos \phi] \cos ^{2} \phi d \phi .
\end{aligned}
$$

Here, the continuity equation (5) is used to obtain the last line. On the other hand, the RHS of Eq. (13) at $z=h$ becomes

$$
\begin{aligned}
\text { [RHS of Eq. (13) at } z=h] & =\left.\nu_{V} \int_{0}^{\pi / 2} \frac{\partial^{2} M_{r}}{\partial z^{2}}\right|_{z=h} \cos \phi d \phi \\
& =\left.\nu_{V} \frac{\partial^{2} M_{0}}{\partial z^{2}}\right|_{z=h} \\
& \approx \nu_{V} \frac{U a \pi^{2}}{2 H^{2}}
\end{aligned}
$$

Now, we assume a single Hadley cell for which equatorward flow just above the surface at $z=h$ is written as $v(\phi, h)=-V_{B} \sin 2 \phi$, where $V_{B}$ is a scale for $v$ at $z=h$. This assumption is based on the numerical results of Yamamoto et al. (2009), showing that meridional circulation forms a single Hadley cell extending to the pole in a quasi-axisymmetric model with large horizontal diffusion, regardless of whether the planetary rotation is slow or fast. As a result, we obtain the following equation from Eqs. (16) and (17):

$$
\frac{8}{15} a \Omega V_{B} \approx \nu_{V} \frac{U a \pi^{2}}{2 H^{2}} .
$$

Dividing Eq. (18) by $a^{2} \Omega^{2}$ and ignoring the numerical factor of $16 / 15 \approx 1$, we obtain

$$
R_{v B} \approx \pi^{2} E_{V} S,
$$

where $R_{v B}=V_{B} /(a \Omega), S=U /(a \Omega)$, and $E_{V} \equiv \nu_{V} /\left(H^{2} \Omega\right)$ is the vertical Ekman number. This equation indicates that the meridional wind just above the bottom surface (represented by $R_{v B}$ ) increases in proportion to the zonal wind at the top (represented by $S$ ).

Next, let us consider Eq. (13) at the top boundary $(z=$ $H)$, where the zonal and poleward components of the horizontal velocity are assumed to be written as $u(\phi, H)$ $=(3 U / 2) \cos \phi$ (i.e., solid-body rotation) and $v(\phi, H)=V_{T}$ $\sin 2 \phi$ (i.e., poleward flow of the assumed single Hadley cell), respectively, where $V_{T}$ is a scale for $v$ at the top. Manipulations similar to those used to obtain Eq. (18) give

$$
-\frac{8}{15}\left(\frac{3}{2} U+a \Omega\right) V_{T} \approx-\nu_{V} \frac{U a \pi^{2}}{2 H^{2}},
$$

and then (with $16 / 15 \approx 1$ and $3 / 2 \approx 1$ )

$$
R_{v T} \approx \pi^{2} E_{V}\left(\frac{S}{1+S}\right),
$$

where $R_{v T}=V_{T} /(a \Omega)$. This equation tells us that the meridional wind at the top (represented by $R_{v T}$ ) increases in proportion to $S$ when $S \ll 1$, whereas it approaches $\pi^{2} E_{V}$ when $S \gg 1$.

\section{b. Thermodynamic equation}

We simplify Eq. (4) for a steady state to obtain an equation that describes the relation among $R_{v B}, R_{v T}$, and $\beta$. The vertical integral of Eq. (4) under $\partial / \partial t=0$, from $z=0$ to $z=H$, divided by $H$ gives

$$
\frac{1}{a H \cos \phi} \int_{0}^{H} \frac{\partial}{\partial \phi}(v \theta \cos \phi) d z=-\frac{1}{H} \int_{0}^{H} \frac{\theta-\theta_{e}}{\tau} d z,
$$

where the continuity equation (5) and vertical boundary conditions (9) and (10) are used. Because of the steady-state assumption, the global mean of $\theta$ should be $\Theta_{0}$, that is, the global mean of $\theta_{e}$. Now, we assume that the vertically averaged potential temperature $\bar{\theta}(\phi)$ has the same meridional dependence as $\theta_{e}$ given by Eq. (8):

$$
\bar{\theta}(\phi) \equiv \frac{1}{H} \int_{0}^{H} \theta(\phi, z) d z \approx \Theta_{0}-\Delta \Theta\left(\sin ^{2} \phi-\frac{1}{3}\right),
$$


where $\Delta \Theta$ is an equator-to-pole difference of the vertically averaged potential temperature. By substituting Eqs. (8) and (23) into Eq. (22), we obtain

$$
\begin{array}{r}
\frac{1}{a H \cos \phi} \int_{0}^{H} \frac{\partial}{\partial \phi}(v \theta \cos \phi) d z \approx \\
-\frac{\left(\Theta_{0} \Delta_{H}-\Delta \Theta\right)}{\tau}\left(\sin ^{2} \phi-\frac{1}{3}\right) .
\end{array}
$$

Here, the LHS of Eq. (24) represents the net heating or cooling in an air column by meridional thermal advection, and the typical scale for the LHS should be proportional to $\Delta \Theta$ and the scale for the meridional wind. On the other hand, the RHS represents the net effect of Newtonian heating and cooling in the air column, and the typical scale for the RHS is $\left(\Theta_{0} \Delta_{H}-\right.$ $\Delta \Theta) / \tau$. Simply comparing the scales of both sides of Eq. (24), we can obtain the following relation:

$$
\frac{\Delta \Theta\left(V_{T}+V_{B}\right)}{2 a} \sim \frac{\left(\Theta_{0} \Delta_{H}-\Delta \Theta\right)}{\tau},
$$

where $\left(V_{T}+V_{B}\right) / 2$ is used for the scale for the meridional wind. Divided by $\Delta \Theta \Omega$, Eq. (25) becomes

$$
\frac{R_{v T}+R_{v B}}{2} \sim \frac{1}{\tau \Omega}\left(\frac{1}{\beta}-1\right),
$$

where $\beta=\Delta \Theta /\left(\Theta_{0} \Delta_{H}\right)$.

\section{c. Meridional momentum equation}

We simplify Eq. (2) for a steady state to obtain an equation that describes the dynamical balance in the meridional momentum equation. Subtracting Eq. (2) with $\partial / \partial t=0$ at $z=h$ from that at $z=H$ gives

$$
\begin{aligned}
& \left.\frac{v(H)}{a} \frac{\partial v}{\partial \phi}\right|_{z=H}-\left.\frac{v(h)}{a} \frac{\partial v}{\partial \phi}\right|_{z=h}+\frac{\left[u(H)^{2}-u(h)^{2}\right] \tan \phi}{a} \\
& \quad+2 \Omega[u(H)-u(h)] \sin \phi \\
& =-\frac{1}{a}\left(\left.\frac{\partial \Phi}{\partial \phi}\right|_{z=H}-\left.\frac{\partial \Phi}{\partial \phi}\right|_{z=h}\right)+\nu_{H}\left\{D_{H}[v(H)]-D_{H}[v(h)]\right\} \\
& \quad+\nu_{\eta}\left(\left.\frac{\partial^{2} v}{\partial z^{2}}\right|_{z=H}-\left.\frac{\partial^{2} v}{\partial z^{2}}\right|_{z=h}\right),
\end{aligned}
$$

where $\phi$ in $u(\phi, z)$ and $v(\phi, z)$ is omitted for readability. The vertical integral from $z=0$ to $z=H$ of partial differentiation of the hydrostatic equation (3) with respect to $\phi$ gives

$$
\begin{aligned}
& \left.\frac{\partial \Phi}{\partial \phi}\right|_{z=H}-\left.\frac{\partial \Phi}{\partial \phi}\right|_{z=0}=\int_{0}^{H} \frac{\partial^{2} \Phi}{\partial \phi \partial z} d z=\frac{g}{\Theta_{0}} \int_{0}^{H} \frac{\partial \theta}{\partial \phi} d z \\
& \quad \approx-\frac{2 g H \Delta \Theta}{\Theta_{0}} \sin \phi \cos \phi,
\end{aligned}
$$

where Eq. (23) is used. Considering the scale of each function in Eq. (27), we can obtain

$$
\begin{aligned}
& \frac{V_{T}^{2}-V_{B}^{2}}{a}+\frac{U^{2}}{a}+2 \Omega U \sim \frac{2 g H \Delta \Theta}{a \Theta_{0}} \\
& -20 \frac{\nu_{H}\left(V_{T}+V_{B}\right)}{a^{2}}+O\left(\nu_{V} \frac{V_{T}+V_{B}}{H^{2}}\right) .
\end{aligned}
$$

Here, Eq. (28) with $\partial \Phi /\left.\partial \phi\right|_{z=h} \approx \partial \Phi /\left.\partial \phi\right|_{z=0}$ and the following scales are used: $u(H) \sim U, u(h) \sim 0, v(H) \sim$ $V_{T}$, and $v(h) \sim V_{B}$. The horizontal diffusion term yields a numerical coefficient of 20 because $v(\phi, H)=V_{T} \sin 2 \phi$ and $v(\phi, h)=-V_{B} \sin 2 \phi$ are assumed [substituting $v=$ $\sin 2 \phi$ into Eq. (7) gives $\left.D_{H}(v)=-20 / a^{2}\right]$. Because we have not specified the vertical structure of $v$, only the order of magnitude of the vertical diffusion term is shown as the third term on the RHS of Eq. (29). Divided by $a \Omega^{2}$, Eq. (29) becomes

$$
\begin{aligned}
& \left(R_{v T}^{2}-R_{v B}^{2}\right)+S^{2}+2 S \sim 2 \beta R_{T}-20 E_{H}\left(R_{v T}+R_{v B}\right) \\
& \quad+O\left[E_{V}\left(R_{v T}+R_{v B}\right)\right]
\end{aligned}
$$

where $R_{T} \equiv g H \Delta_{H} /\left(a^{2} \Omega^{2}\right)$ is the external thermal Rossby number and $E_{H} \equiv \nu_{H} /\left(a^{2} \Omega\right)$ is the horizontal Ekman number.

Note that the Gierasch mechanism requires the relaxation time for horizontal diffusion to be much shorter than both the turnover time of meridional circulation and the relaxation time for vertical diffusion. In the nondimensional numbers, these conditions are written as $E_{H} \gg R_{v B}$ and $E_{H} \gg E_{V}$, where $R_{v B}$ is used for the reciprocal of the nondimensional turnover time because $R_{v B}>R_{v T}$ [Eqs. (19) and (21)]. The present study is also based on these conditions. Multiplying the inequalities by $\left(R_{v T}+R_{v B}\right)$, we obtain the following relations: $E_{H}\left(R_{v T}+R_{v B}\right) \gg R_{v B}\left(R_{v T}+R_{v B}\right)$ $>\left|R_{v T}^{2}-R_{v B}^{2}\right|$ and $E_{H}\left(R_{v T}+R_{v B}\right) \gg E_{V}\left(R_{v T}+R_{v B}\right)$. Therefore, we can ignore the advection term $\left(R_{v T}^{2}-R_{v B}^{2}\right)$ and the vertical diffusion term $O\left[E_{V}\left(R_{v T}+R_{v B}\right)\right]$ in Eq. (30) to obtain

$$
S^{2}+2 S \sim 2 \beta R_{T}-20 E_{H}\left(R_{v T}+R_{v B}\right) .
$$

Here, the first term on the LHS of Eq. (31) is the metric term and the second is the Coriolis term, whereas the first term on the RHS is the term representing the pressure gradient and the second is the horizontal diffusion term.

\section{d. Quintic equation for superrotation strength}

Now, let us regard Eqs. (19), (21), (26), and (31) as a set of algebraic equations that characterize the general circulation of planetary atmospheres under the assumptions given in the previous subsections. Namely, the four equations are simultaneous equations whose unknowns are $S, R_{v B}, R_{v T}$, and $\beta$. Eliminating $R_{v B}$, $R_{v T}$, and $\beta$ from the equations, we obtain the following 
quintic equation for $S$ :

$$
\left[S^{2}+2 S+B S\left(\frac{2+S}{1+S}\right)\right]\left[\frac{A S}{2}\left(\frac{2+S}{1+S}\right)+1\right]=2 R_{T},
$$

where $A \equiv \pi^{2} \tau \Omega E_{V}, B \equiv 20 \pi^{2} E_{H} E_{V}$, and $R_{T}$ are positive constants that consist of external parameters. Note that $A$ is proportional to the ratio of the radiative relaxation time to the timescale for the vertical eddy diffusion of momentum because $A$ can be written as $\pi^{2}$ $\tau\left(\nu_{V} / H^{2}\right)$, and that $B$ is proportional to the square of the ratio of the planetary rotation period to the geometric mean of the timescales for the horizontal and vertical eddy diffusion of momentum because $B$ can be written as $20 \pi^{2}\left[\Omega^{-1} \sqrt{\left(\nu_{H} / a^{2}\right)\left(\nu_{V} / H^{2}\right)}\right]^{2}$.

This quintic equation has only one positive solution (see Appendix for the demonstration), which is physically valid. The other solutions are complex or negative. Complex solutions have no physical meaning in this context. Negative solutions mean that the zonal wind rotates against the sense of planetary rotation with an indirect meridional circulation, which is an unrealistic situation. Hereafter, we focus on the positive solution.

The quintic equation (32) shows that the superrotation strength $S$ depends only on $A, B$, and $R_{T}$. Because of Eqs. (19) and (21), $R_{v B}$ and $R_{v T}$ depend on $S$ and $E_{V}$. In other words, $R_{v B}$ and $R_{v T}$ depend on $A, B, R_{T}$, and $E_{V}$. On the other hand, $\beta$ does not explicitly depend on $E_{V}$, because the following relation between $\beta$ and $S$ can be obtained by substituting Eqs. (19) and (21) into Eq. (26):

$$
\beta=\left[\frac{A S}{2}\left(\frac{2+S}{1+S}\right)+1\right]^{-1} .
$$

In other words, $\beta$ depends only on $A, B$, and $R_{T}$.

\subsection{A further simplification}

In this subsection, we simplify the quintic equation (32) to a cubic equation. Then, we present a simpler expression of the solution depending on the parameters and investigate the parameter dependence of the dominant dynamical balance in the meridional component of the momentum equation (2), such as a geostrophic balance and a cyclostrophic balance.

We approximate the quintic equation (32), which is not analytically solvable, to the following cubic equation, which is analytically solvable. By assuming that $(2+S) /(1+S) \approx C$ (a constant), which is valid because $1<(2+S) /(1+S)<2$ for $S>0$, we obtain

$$
\left(S^{2}+2 S+B C S\right)\left(\frac{A C S}{2}+1\right) \approx 2 R_{T},
$$

or

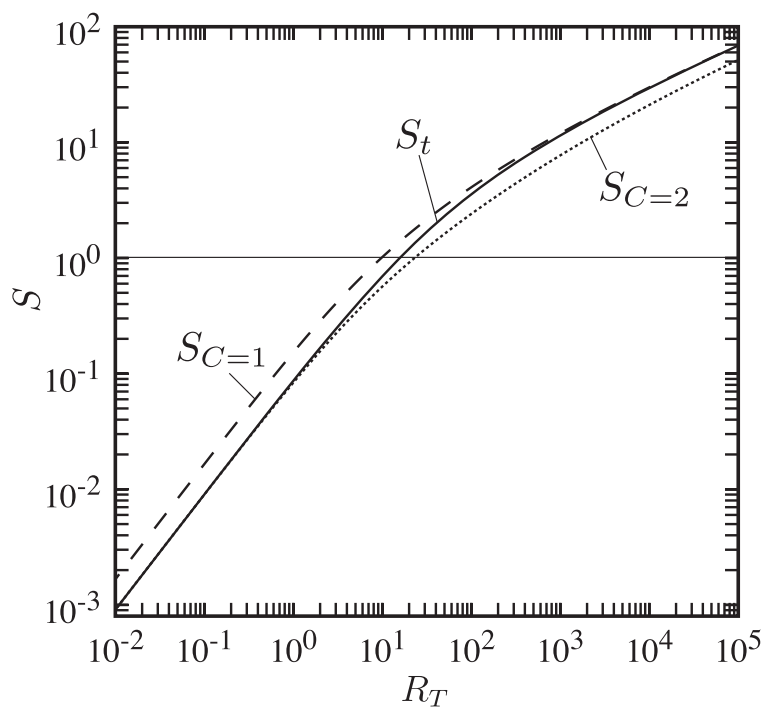

Fig. 1. Positive solution of the quintic equation (32) (solid), the positive solution of the cubic equation (35) with $C=1$ (dashed), and that with $C=2$ (dotted) for $A=1, B=10$, and $10^{-2} \leq R_{T} \leq 10^{5}$ (abscissa). The solution of the quintic equation is obtained by Newton's method, whereas the solution of the cubic equation is obtained by Cardano's formula. The horizontal line indicates $S=1$.

$$
\frac{A C}{2} S^{3}+\left(1+A C+\frac{A B C^{2}}{2}\right) S^{2}+(2+B C) S \approx 2 R_{T}
$$

Since $A, B, C$, and $R_{T}$ are positive constants, Eq. (35) also has only one positive solution.

Now, let us denote the solution of the quintic equation (32) by $S_{t}$, the solution of the cubic equation (35) with $C=1$ by $S_{C=1}$, and that with $C=2$ by $S_{C=2}$. Figure 1 shows the dependence on $R_{T}$ of $S_{t}$ (solid), $S_{C=1}$ (dashed), and $S_{C=2}$ (dotted) for $10^{-2} \leq R_{T} \leq 10^{5}$ with $A=1$ and $B=10$ as an example. Here, Newton's method is used to obtain the values of $S_{t}$ and Cardano's formula is used to obtain the values of $S_{C=1}$ and $S_{C=2}$. The figure evidently shows the validity of $S_{t} \approx S_{C=1}$ for $S_{t} \gg 1$ and $S_{t} \approx S_{C=2}$ for $S_{t} \ll 1$.

Because the solution obtained by Cardano's formula is too complicated for analytical treatment, we consider some cases of dominant balances of Eq. (35). That is, we consider the situations where one of the three terms on the LHS of Eq. (35) is much larger than the others and nearly equal to $2 R_{T}{ }^{2}$ By assuming these three situations separately, we obtain three simpler expressions of the solution $\left(S_{i} ; i=1,2\right.$, and 3$)$ as follows: 
Table 1. Simple expressions of the approximated solutions (37) and their dominant dynamical balance (square brackets). Note that $A=\pi^{2} \tau \Omega E_{V}$ and $B=20 \pi^{2} E_{H} E_{V}$, and $C=1$ should be used if $S>1$ and $C=2$ should be used if $S \leq 1$.

\begin{tabular}{|c|c|c|c|c|c|c|c|}
\hline & $B$ & $A(A B)$ & $S_{1}$ & $S_{2}$ & $S_{3}$ & $R_{T 1}$ & $R_{T 2}$ \\
\hline (a) & \multirow{2}{*}{$B \ll 1$} & $A \ll 1$ & \multirow{2}{*}{$R_{T}[\mathrm{G} 1]$} & $\sqrt{2 R_{T}}[\mathrm{C} 1]$ & \multirow{4}{*}{$\left(\frac{4 R_{T}}{A C}\right)^{\frac{1}{3}}[\mathrm{C} 0]$} & 2 & $\frac{2}{A^{2} C^{2}}$ \\
\hline (b) & & $A \gg 1$ & & $\sqrt{\frac{2 R_{T}}{A C}}[\mathrm{G} 0]$ & & $\frac{2}{A C}$ & $2 A C$ \\
\hline (c) & \multirow{2}{*}{$B \gg 1$} & $A B \ll 1$ & \multirow{2}{*}{$\frac{2 R_{T}}{B C}[\mathrm{H} 1]$} & $\sqrt{2 R_{T}}[\mathrm{C} 1]$ & & $\frac{B^{2} C^{2}}{2}$ & $\frac{2}{A^{2} C^{2}}$ \\
\hline (d) & & $A B \gg 1$ & & $\sqrt{\frac{4 R_{T}}{A B C^{2}}}[\mathrm{H} 0]$ & & $\frac{B}{A}$ & $\frac{A B^{3} C^{4}}{4}$ \\
\hline
\end{tabular}

$$
\begin{aligned}
& S_{1} \equiv \frac{2 R_{T}}{2+B C}, \quad S_{2} \equiv \sqrt{\frac{2 R_{T}}{1+A C+0.5 A B C^{2}}}, \\
& \text { and } \quad S_{3} \equiv\left(\frac{4 R_{T}}{A C}\right)^{\frac{1}{3}} .
\end{aligned}
$$

For the assumed situations to be valid, the other two terms evaluated using $S_{i}$ must be much less than $2 R_{T}$. Conversely, $S_{t}$ can be approximated by $S_{i}$ when the other two terms evaluated using $S_{i}$ are much less than $2 R_{T}$ :

$$
S_{t} \approx\left\{\begin{array}{l}
S_{1} \text { for } R_{T} \ll R_{T 1} \\
S_{2} \text { for } R_{T 1} \ll R_{T} \ll R_{T 2} ; \\
S_{3} \text { for } R_{T 2} \ll R_{T}
\end{array}\right.
$$

where

$$
\begin{aligned}
& R_{T 1} \equiv \frac{(2+B C)^{2}}{2\left(1+A C+0.5 A B C^{2}\right)} \quad \text { and } \\
& R_{T 2} \equiv \frac{2\left(1+A C+0.5 A B C^{2}\right)^{3}}{A^{2} C^{2}}
\end{aligned}
$$

The above formulation clarifies the dominant dependence of $S_{t}$ on $A, B, C$, and $R_{T}$. To investigate the dominant dynamical balance, however, formulations (36) and (38) are still complicated.

For the next step, we approximate $S_{1}, S_{2}, R_{T 1}$, and $R_{T 2}$ by considering the orders of magnitude of $A, B$, and $A B$. The denominator of $S_{1}$ and the numerator of $R_{T 1}$ include $(2+B C)$, which can be approximated to 2 for $B$ $\ll 1$ and to $B C$ for $B \gg 1$. (Note that $C \sim 1$.) In addition, the denominators of $S_{2}$ and $R_{T 1}$ and the numerator of $R_{T 2}$ include $\left(1+A C+0.5 A B C^{2}\right)$, in

${ }^{2}$ Every term on the LHS of Eq. (35) is positive because $A, B$, and $C$ are positive and we have focused on the positive solution $(S>0)$. Therefore, there is no dominant balance of Eq. (35) without the RHS. which $A \gg A B$ when $B \ll 1$ and $A \ll A B$ when $B \gg 1$. For these relationships, when $B \ll 1$, we can approximate $\left(1+A C+0.5 A B C^{2}\right)$ to 1 for $A \ll 1$ and to $A C$ for $A \gg 1$. When $B \gg 1$, on the other hand, we can approximate $\left(1+A C+0.5 A B C^{2}\right)$ to 1 for $A B \ll 1$ and to $0.5 A B C^{2}$ for $A B \gg 1$. These relationships allow us to approximate $S_{1}, S_{2}, R_{T 1}$, and $R_{T 2}$ to the formulations shown in Table 1 for the four cases indicated by (a)-(d). The symbols in the square brackets $(\mathrm{C} 1, \mathrm{C} 0, \mathrm{G} 1, \mathrm{G} 0$, $\mathrm{H} 1$, and $\mathrm{H} 0$ ) in Table 1 indicate types of the dominant dynamical balance, which we explain in the next subsection.

In Fig. 2, the solid curves show the dependence of $S_{t}$, the solution of the quintic equation (32), on $R_{T}$ for (a) $A=10^{-2}, B=10^{-2}$, (b) $A=10^{2}, B=10^{-2}$, (c) $A=10^{-3}$, $B=10^{2}$, and (d) $A=10^{2}, B=10$. Note that the values of $A$ and $B$ for each small letter label (a), (b), (c), or (d) satisfy the inequalities labeled by the same letter given in Table 1. The dashed lines show the dependence of the simple expressions of $S_{i}$ as obtained above (given in Table 1) on $R_{T}$. Here, $C=1$ is used for $S_{i}>1$, and $C=$ 2 is used for $S_{i} \leq 1$. The values of $S_{1}\left(R_{T 1}\right)$ and $S_{2}\left(R_{T 2}\right)$ obtained from the simple expressions are also shown. The dominant dependence of $S_{t}$ on $R_{T}$ (solid curve) changes from $S_{t} \propto R_{T}$ to $S_{t} \propto \sqrt{R_{T}}$ to $S_{t} \propto R_{T}^{1 / 3}$, as predicted by the approximated solutions (36). In addition, the simple expressions given in Table 1 (dashed lines) approximate $S_{t}$ well, except around $R_{T}=$ $R_{T 1}$ or $R_{T 2}$.

\subsection{Dominant dynamical balance}

Depending on $A, B$, and $R_{T}$, there are six types of simple expressions of the solutions for $S_{t}$, as listed in Table 1. This fact implies that there are six types of typical dynamical balances. The dynamical balance becomes apparent in the following form of the 

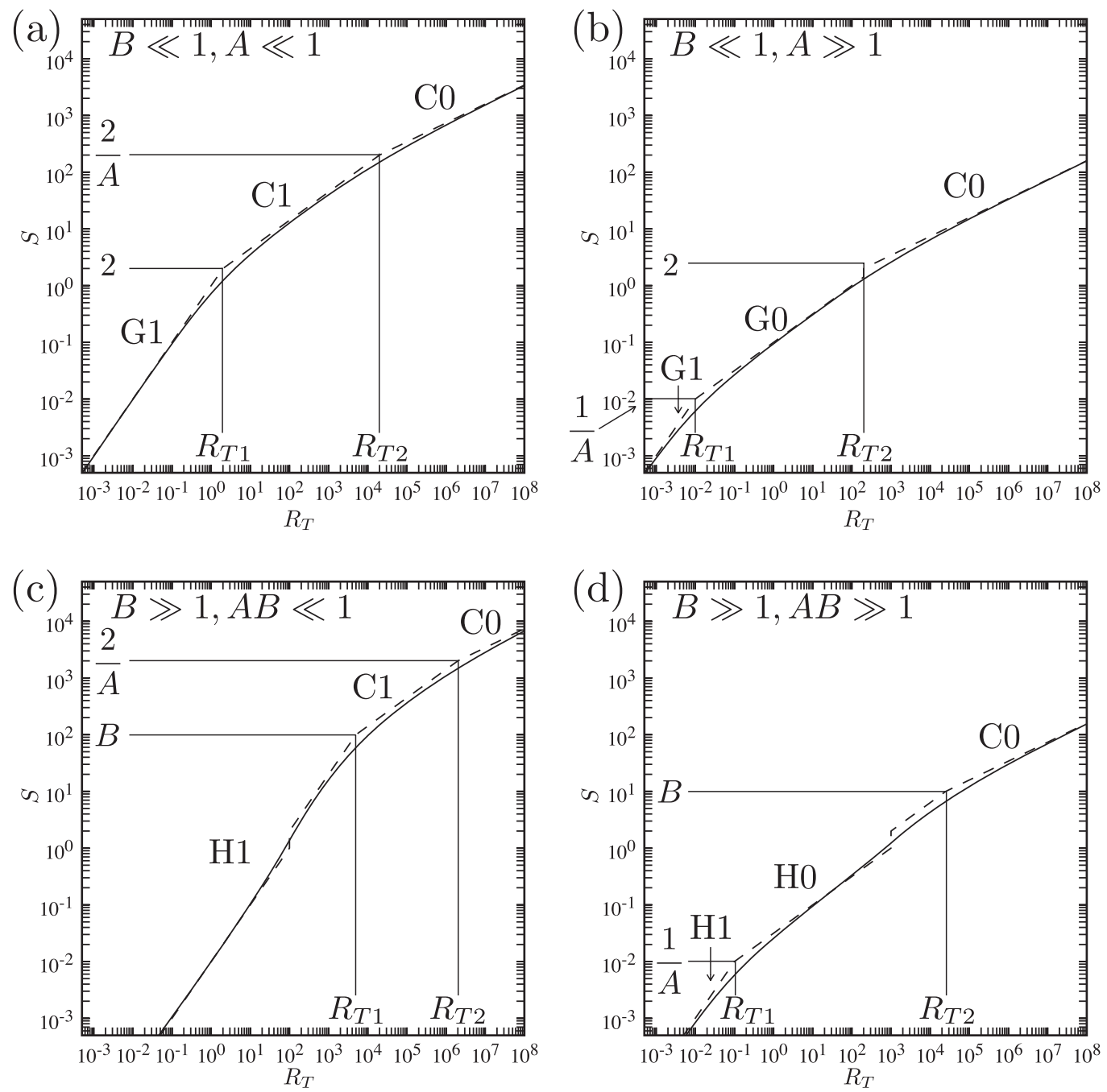

Fig. 2. Solution of the quintic equation (32) (solid) and simple expressions shown in Table 1 (dashed) as functions of $R_{T}$ (abscissa) for (a) $A=10^{-2}, B=10^{-2}$, (b) $A=10^{2}, B=10^{-2}$, (c) $A=10^{-3}, B=10^{2}$, and (d) $A=10^{2}, B=10$. The vertical lines indicate the locations of $R_{T 1}$ (left) and $R_{T 2}$ (right). The horizontal lines indicate $S_{1}\left(R_{T 1}\right)$ and $S_{2}\left(R_{T 2}\right)$. The types of the dominant dynamical balance are indicated by $\mathrm{C} 1, \mathrm{C} 0, \mathrm{G} 1, \mathrm{G} 0, \mathrm{H} 1$, and $\mathrm{H} 0$, as given in Table 1 .

meridional momentum equation obtained from Eq. (34):

$$
\underbrace{S^{2}}_{\text {I }}+\underbrace{2 S}_{\text {II }}+\underbrace{B C S}_{\text {III }} \approx \underbrace{2 R_{T} \beta}_{\text {IV }}
$$

where

$$
\beta=\left(\frac{A C S}{2}+1\right)^{-1}
$$

which is obtained from Eq. (33) with $(2+S) /(1+S)=C$. Term I represents the metric term, II the Coriolis term, III the horizontal diffusion term acting on the meridional wind, and IV the pressure gradient term. Let us consider the situations where one of the three terms on the LHS of Eq. (39) almost balances with term IV. That is, the dominant balance is between term IV

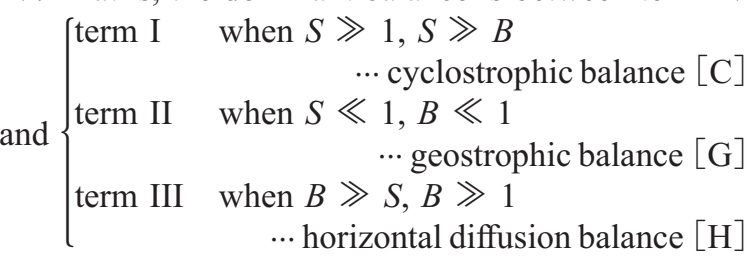
Here, the horizontal diffusion balance means the balance between the horizontal diffusion and the 
pressure gradient force, and here, we do not consider three- and four-way balances, such as a gradient wind balance.

Furthermore, $\beta$ can be approximated for the following two cases:

$$
\beta \approx\left\{\begin{array}{lll}
1 & \text { when } A S \ll 1 & \cdots[1] \\
(0.5 A C S)^{-1} \text { when } A S \gg 1 & \cdots[0]
\end{array}\right.
$$

The former case [1] means that the equator-to-pole difference of the potential temperature $\Delta \Theta$ is almost equal to that of the basis potential temperature for Newtonian heating and cooling $\Theta_{0} \Delta_{H}$, and the magnitude of pressure gradient term IV is given by the external parameter $R_{T}$. On the other hand, the latter case [0] means that $\Delta \Theta$ is much smaller than $\Theta_{0} \Delta_{H}$, and the magnitude of pressure gradient term IV is internally determined because of the dependence on $S$. Consequently, the typical dynamical balance in Eq. (39) can be classified into the six types, which are the combinations of $[\mathrm{C}, \mathrm{G}, \mathrm{H}]$ and $[1,0]$. For example, $\mathrm{C} 1$ indicates the cyclostrophic balance with $\beta \approx 1$, and G0 indicates the geostrophic balance with $\beta \approx$ $(0.5 A C S)^{-1} \ll 1$. The classifications are also included in Table 1 and Fig. 2 to show the dependence on $A, B$, and $R_{T}$.

\section{Numerical solutions}

\subsection{Numerical procedure}

Numerical solutions of governing equations (1)-(11) are obtained by time-integrations of a discretized set of the equations. For spatial discretization, we use a spectral transform method in the meridional direction and a central difference method in the vertical direction. The truncation order of the Legendre polynomial is 85 (64 grid points at the Gaussian latitudes from the equator to the pole) and the number of layers in the vertical direction is 50, with a uniform depth. The equations are integrated using the classical fourth-order Runge-Kutta method from an initial state of rest with constant potential temperature $\Theta_{0}$ until a steady state or statistically steady state is achieved. The time step is an appropriate value between $6 \mathrm{~s}$ and 5400 $\mathrm{s}$ depending on the parameter values; this is because a smaller $R_{T}$ value requires a shorter time step for numerical stability.

Numerical solutions are obtained for $R_{T}=10^{n}$, where $n=-2,-1,0, \ldots, 5$, with four combinations of the values of $A=\pi^{2} \tau \Omega E_{V}$ and $B=20 \pi^{2} E_{H} E_{V}$ with $E_{V}$ fixed at $10^{-3}$, as listed in Table 2 (a)-(d). Note that the values of $A$ and $B$ for each small letter label (a), (b), (c), or (d) in Table 2 satisfy the inequalities labeled by the same letter given in Table 1 . The values of $A$ and $B$
Table 2. Values of $A, B$, and $E_{V}$ for the numerical experiments (a)-(d) and (d'). The values of $A$ and $B$ for each label satisfy the inequalities labeled by the same letter in Table 1. Here, $A=\pi^{2} \tau \Omega E_{V}$ and $B=20 \pi^{2} E_{H} E_{V}$, and the used values of $\tau \Omega$ and $E_{H}$ are also listed.

\begin{tabular}{cccc|cc}
\hline & $A$ & $B$ & $E_{V}$ & $\tau \Omega$ & $E_{H}$ \\
\hline (a) & $\pi^{2} \times 10^{-2}$ & $2 \pi^{2} \times 10^{-2}$ & $10^{-3}$ & 10 & 1 \\
(b) & $\pi^{2}$ & $2 \pi^{2} \times 10^{-2}$ & $10^{-3}$ & $10^{3}$ & 1 \\
(c) & $\pi^{2} \times 10^{-3}$ & $2 \pi^{2}$ & $10^{-3}$ & 1 & $10^{2}$ \\
(d) & $\pi^{2} \times 10^{-1}$ & $2 \pi^{2}$ & $10^{-3}$ & $10^{2}$ & $10^{2}$ \\
(d') & $\pi^{2} \times 10^{-1}$ & $2 \pi^{2}$ & $10^{-2}$ & 10 & 10 \\
\hline
\end{tabular}

differ from those used in Fig. 2 in order to avoid difficulty in obtaining numerical solutions. Finer resolution and shorter time steps are needed for the values used in Fig. 2. Here, $E_{V}$ is fixed because $R_{v B}$ and $R_{v T}$ explicitly depend on $E_{V}$, as given by Eqs. (19) and (21). The dependence on $E_{V}$ is verified at the end of this section. The other nondimensional parameters are fixed at $P_{r V} \equiv \nu_{V} / \kappa_{V}=1$ and $\Delta_{H}=0.1$. Hereafter, the numerical results for each of the four combinations are labeled by (a)-(d) and the value of $R_{T}$; for example, "(a) $R_{T}=10^{2}$ " means the numerical result obtained with $A=$ $\pi^{2} \times 10^{-2}, B=2 \pi^{2} \times 10^{-2}, E_{V}=10^{-3}$, and $R_{T}=10^{2}$. For numerical time-integrations, we use the following dimensional parameter values of Venus: $a=6.05 \times$ $10^{6} \mathrm{~m}, H=5 \times 10^{4} \mathrm{~m}, g=8.84 \mathrm{~m} \mathrm{~s}^{-2}$, and $\Theta_{0}=500 \mathrm{~K}$, where $H$ and $\Theta_{0}$ are just rough estimations. According to these values and the nondimensional parameter values, the values of the other dimensional parameters $\left(\Omega, \nu_{H}, \nu_{V}, \kappa_{V}\right.$, and $\left.\tau\right)$ are determined uniquely.

\subsection{Description of numerical solutions}

Steady-state solutions are obtained for (a) $R_{T} \leq 10^{\circ}$ and $R_{T}=10^{5}$, (b) $R_{T} \leq 10^{3}$, (c) $R_{T} \leq 10^{2}$ and $R_{T} \geq$ $10^{4}$, and (d) $R_{T} \leq 10^{3}$. For the other cases, the solutions achieve statistically steady states, and the solutions time-averaged over more than ten periods of the oscillation are used for analyses in this section.

Figure 3 shows the nondimensional zonal wind $u(\phi, z) /(a \Omega)$ of the steady-state or time-averaged solutions. The contour interval is one tenth of the maximum value, which is shown above each panel. Aside from (a) $R_{T}=10^{5}$ and (c) $R_{T} \geq 10^{4}$, a "deep" prograde equatorial jet exists, and the atmosphere is in a nearly solid-body rotation. The maximum value of $u /(a \Omega)$ increases as $R_{T}$ increases. In contrast, a prograde equatorial jet is "shallow" (i.e., confined to a thin upper layer) and relatively slow for (a) $R_{T}=10^{5}$ and (c) $R_{T} \geq$ $10^{4}$. 


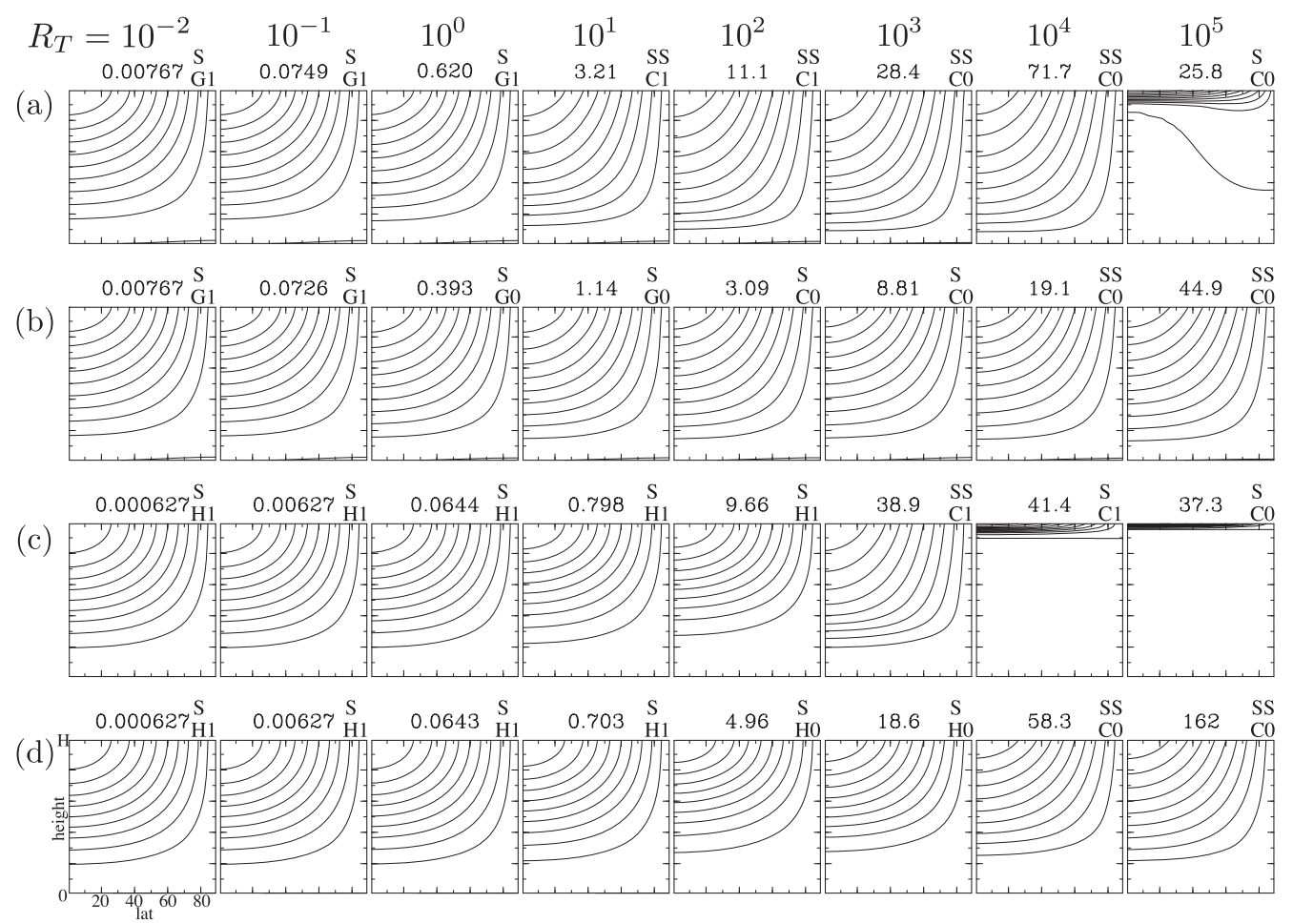

Fig. 3. Nondimensional zonal wind $u(\phi, z) /(a \Omega)$ in steady or statistically steady states (indicated by S and SS, respectively) obtained by time-integrations from a state of rest with a constant potential temperature for (a) $A=\pi^{2} \times 10^{-2}, B=2 \pi^{2} \times$ $10^{-2}$, (b) $A=\pi^{2}, B=2 \pi^{2} \times 10^{-2}$, (c) $A=\pi^{2} \times 10^{-3}, B=2 \pi^{2}$, and (d) $A=\pi^{2} \times 10^{-1}, B=2 \pi^{2}$. The value of $R_{T}$ is shown at the top. The maximum value in each case is shown above the panel. The types of the dominant dynamical balance predicted by the theoretical model are also shown. Contour interval is one tenth of the maximum value.

Figure 4 shows the nondimensional meridional streamfunction $\Psi(\phi, z) /(a H \Omega)$, where $v=-\partial \Psi / \partial z$ and $w=(a \cos \phi)^{-1} \partial(\Psi \cos \phi) / \partial \phi$. The contour interval is one tenth of the maximum value, which is shown above each panel. For the steady-state solutions, the meridional circulation has a one-cell structure, whereas for the statistically steady-state solutions, the meridional circulation has an inclined direct cell with convectional cells, such as (a) $R_{T}=10^{1}$, or that with indirect cells, such as (a) $R_{T}=10^{2}$. The structure of a one-cell meridional circulation gradually becomes asymmetric with respect to the mid-level of $z=H / 2$ and becomes inclined as $R_{T}$ increases. The strength of the circulation increases as $R_{T}$ increases, and the shallowjet solutions have markedly strong circulations.

Figure 5 shows the nondimensional potential temperature $\theta(\phi, z) / \Theta_{0}$. The contour interval is 0.01 for all panels. When $R_{T}$ is small, $\theta$ is almost equal to $\theta_{e}$. In contrast, when $R_{T}$ is large, the latitudinal gradient of $\theta$ is smaller than that of $\theta_{e}$. These facts are consistent with the strength of the meridional circulation.
Namely, the meridional circulation decreases the latitudinal temperature gradient by thermal advection.

\subsection{Multiple equilibrium states}

For (a) $R_{T}=10^{5}$ and (c) $R_{T}=10^{4}$, another equilibrium state, which has a strong deep jet and a weak meridional circulation as shown in Fig. 6, is achieved by time-integrations from the statistically steady-state solutions of (a) $R_{T}=10^{4}$ and (c) $R_{T}=10^{3}$, respectively. The existence of both shallow-jet and deep-jet solutions for the same external conditions indicates that our system has at least two stable equilibrium states, at least when the parameters are (a) $R_{T}=10^{5}$ or (c) $R_{T}=10^{4}$. We also performed time-integrations for (c) $R_{T}=10^{5}$ from the deep-jet solution of (c) $R_{T}=10^{4}$ obtained above, but we obtained the same shallow-jet solution described in the previous subsection without obtaining multiple equilibrium states.

Such multiple equilibrium states were first found by Matsuda $(1980,1982)$ in a highly truncated spectral model. Kido and Wakata $(2008,2009)$ also obtained 


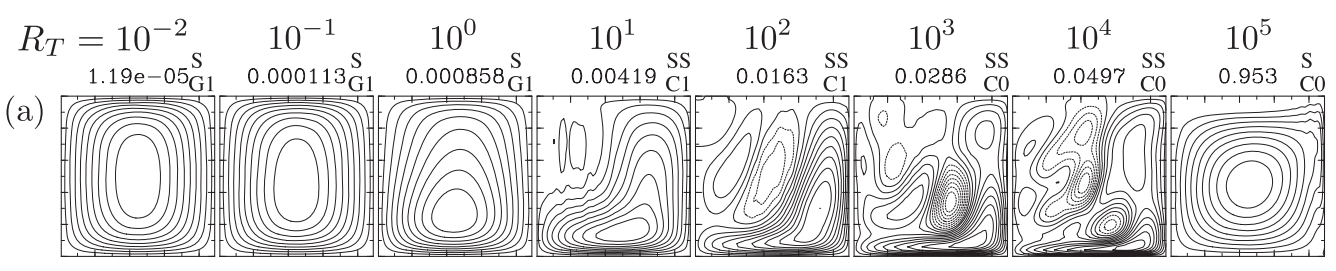

(b)

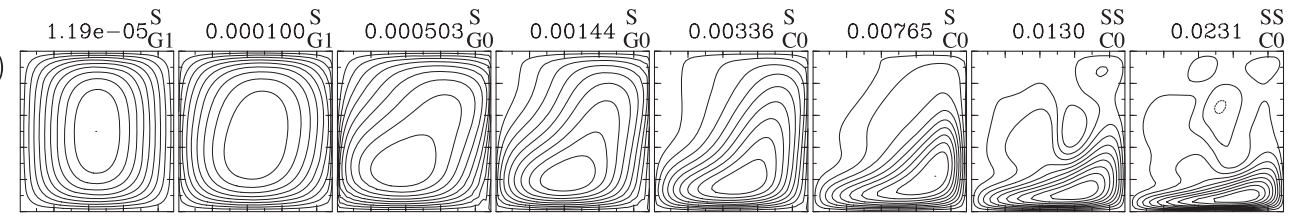

(c)

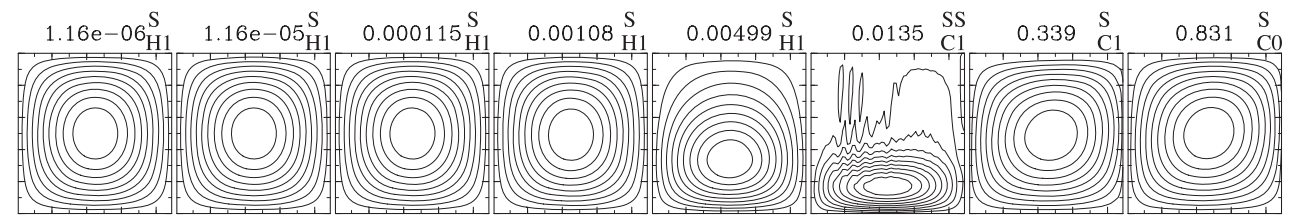

(d)

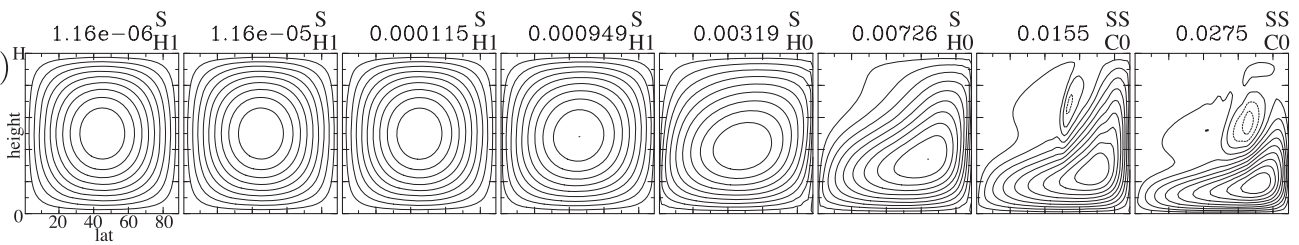

Fig. 4. Same as Fig. 3 but for nondimensional meridional streamfunction $\Psi(\phi, z) /(a H \Omega)$, where $v=-\partial \Psi / \partial z$ and $w=(a$ $\cos \phi)^{-1} \partial(\Psi \cos \phi) / \partial \phi$.

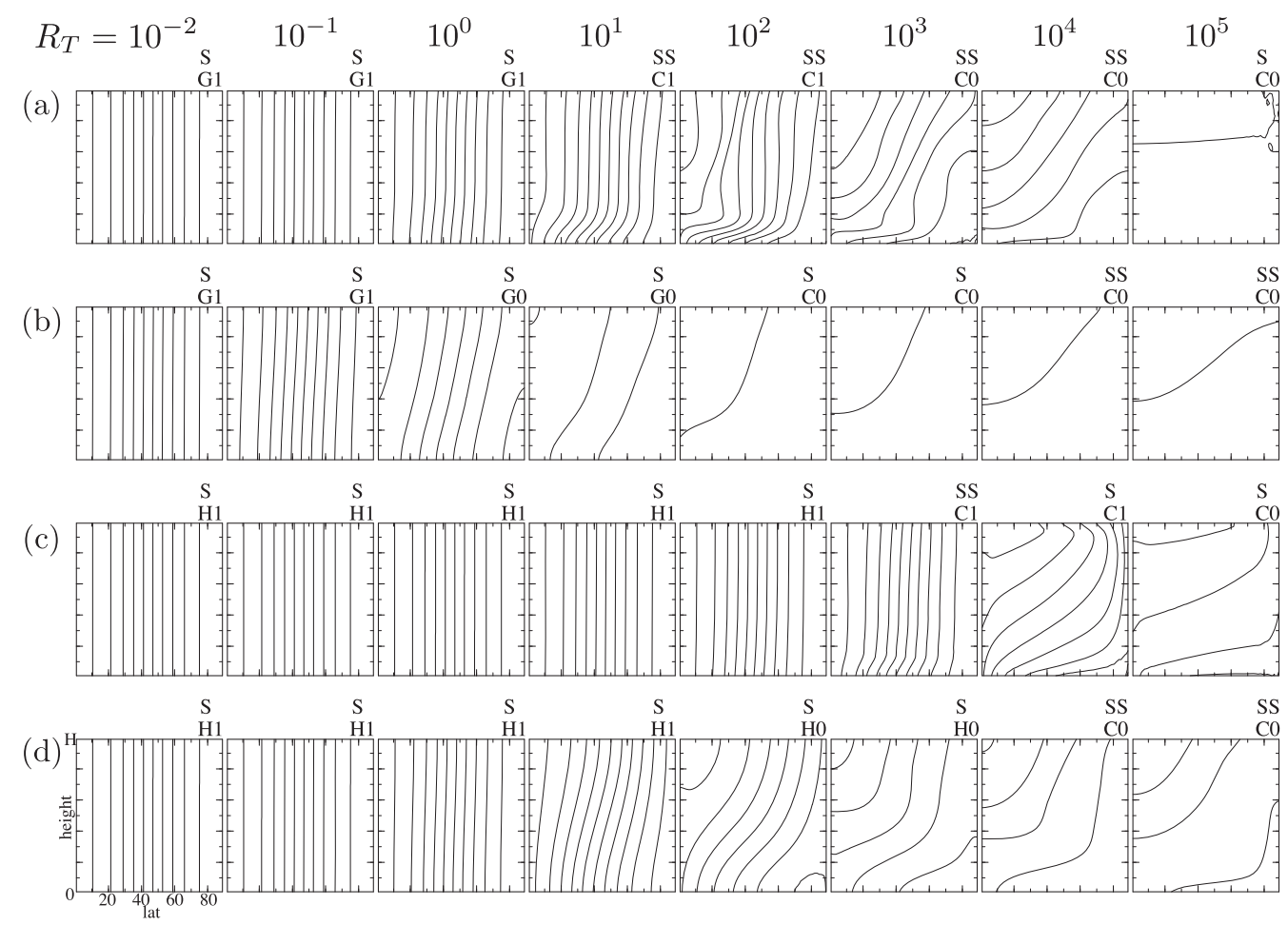

Fig. 5. Same as Fig. 3 but for nondimensional potential temperature $\theta(\phi, z) / \Theta_{0}$. Contour interval is 0.01 . 


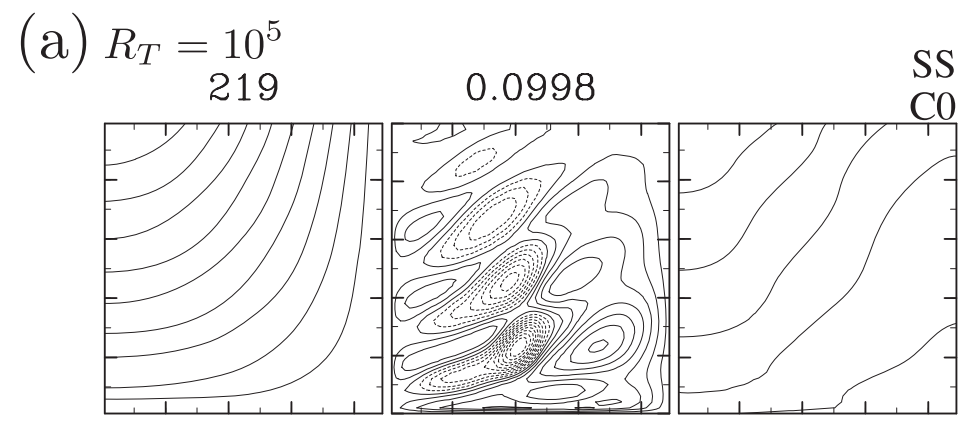

(c) $R_{T}=10^{4}$

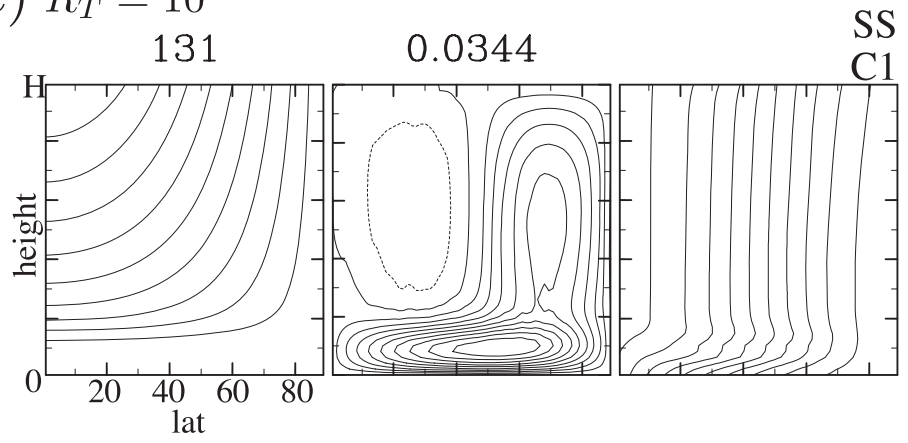

Fig. 6. Nondimensional zonal wind (left), nondimensional meridional streamfunction (middle), and nondimensional potential temperature (right) in statistically steady states for (a) $A=\pi^{2} \times 10^{-2}, B=2 \pi^{2} \times 10^{-2}, R_{T}=10^{5}$ and (c) $A=\pi^{2}$ $\times 10^{-3}, B=2 \pi^{2}, R_{T}=10^{4}$. For (a) $R_{T}=10^{5}$, the statistically steady state is obtained by time-integrations from the solution for (a) $R_{T}=10^{4}$. For (c) $R_{T}=10^{4}$, the statistically steady state is obtained by time-integrations from the solution for (c) $R_{T}=10^{3}$. Contour intervals are same as Figs. 3-5.

similar multiple equilibrium states in a Venus-like GCM.

\subsection{Comparison with theoretical solutions}

To compare the numerical results with $S_{t}$, which is the positive solution of the quintic equation (32), we introduce a measure of the superrotation strength $S_{n}$ for the obtained numerical solutions, defined as

$$
S_{n} \equiv \frac{1}{a \Omega} \sum_{j=1}^{J} u\left(\phi_{j}, z_{K}\right) \cos \phi_{j} \Delta \phi_{j}
$$

Here, $\phi_{1}, \phi_{2}, \phi_{3}, \ldots, \phi_{J}(J=64)$ are the Gaussian latitudes from the equator to the pole, $z_{K}$ is the altitude of the top layer, $\Delta \phi_{j} \equiv\left(\phi_{j+1}-\phi_{j-1}\right) / 2$, where $j=2,3$, $4, \ldots, J-1, \Delta \phi_{1} \equiv\left(\phi_{2}+\phi_{1}\right) / 2$, and $\Delta \phi_{J} \equiv\left(\pi-\phi_{J}-\right.$ $\left.\phi_{J-1}\right) / 2$. Figure 7 shows the dependence of $S_{n}$ on $R_{T}$ for (a)-(d). The circles indicate deep-jet solutions (solid symbols for steady states and open ones for statistically steady states), the crosses indicate shallowjet solutions, the solid curves denote the theoretical solutions $S_{t}$, the two vertical lines on each panel indicate $R_{T 1}$ (left) and $R_{T 2}$ (right), and the types of dominant dynamical balance described in the previous section are indicated by $\mathrm{C} 1, \mathrm{C} 0, \mathrm{G} 1, \mathrm{G} 0, \mathrm{H} 1$, and $\mathrm{H} 0$. The dotted curves denote Matsuda's (1980) estimates $S_{M}$, which we discuss in Section 5.4. For the statistically steady-state solutions, $S_{n}$ oscillates with an amplitude less than $3 \%$ of the time average of $S_{n}$ (variable ranges are not shown).

Within the parameter range we studied, $S_{t}$ agrees well with $S_{n}$ for deep-jet solutions. The range of the relative error defined as $e_{r} \equiv\left(S_{t}-S_{n}\right) / S_{n}$ is $-0.34 \leq$ $e_{r} \leq 0.38$ for the steady-state solutions and $-0.48 \leq$ $e_{r} \leq 0.23$ for the statistically steady-state solutions. Compared with the parameter range covering many orders of magnitude, a relative error of less than $50 \%$ is very small. In addition, there is no significant difference in $e_{r}$ between the steady-state solutions and the statistically steady-state solutions. This implies that our estimation of superrotation strength is valid not only for the steady-state solutions but also for the statistically steady-state solutions, even though our theoretical model is based on the steady-state assumption. 
(a)

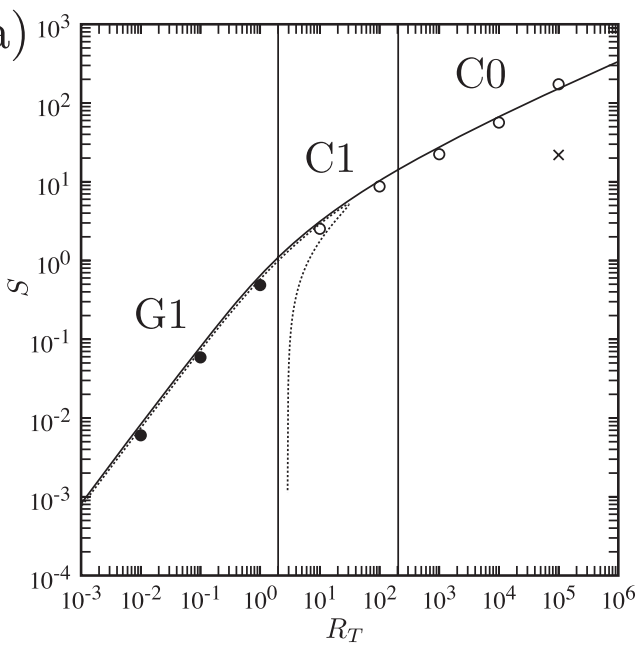

(c)

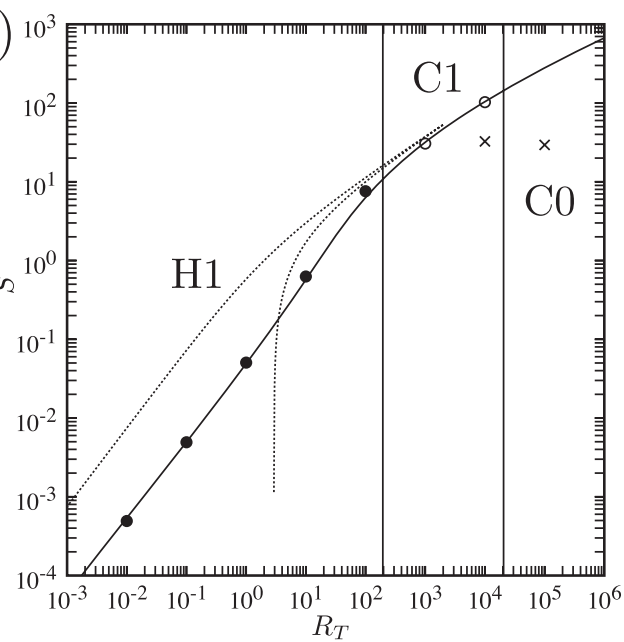

(b)

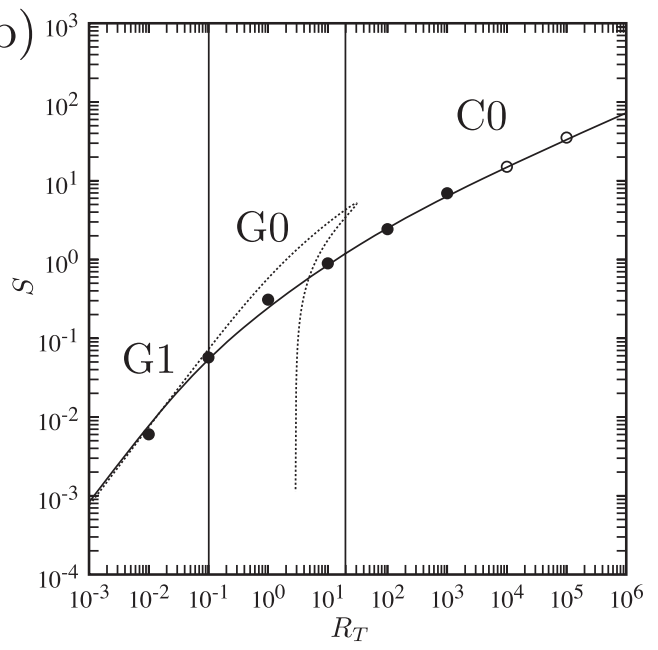

(d)

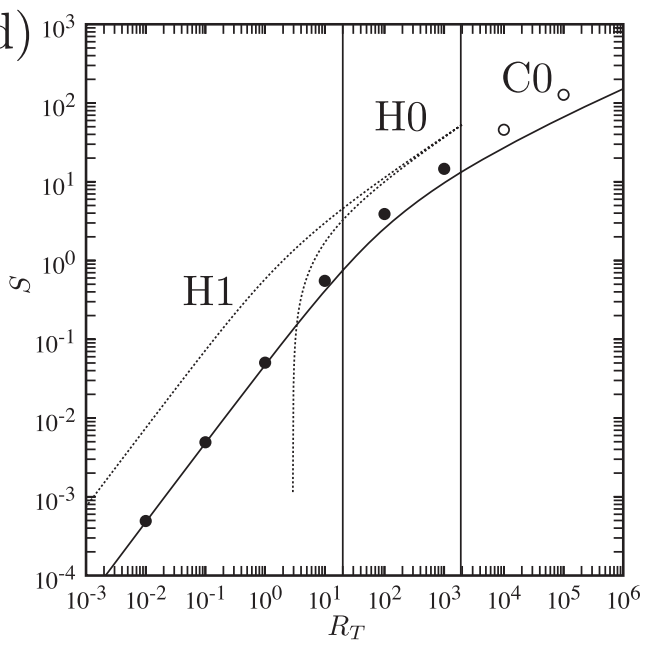

Fig. 7. Superrotation strength $S_{n}$ obtained from the numerical deep-jet solutions in steady (solid circle) and statistically steady (open circle) states and from the shallow-jet solutions (cross symbol) plotted against $R_{T}$ (abscissa) for (a) $A=\pi^{2} \times$ $10^{-2}, B=2 \pi^{2} \times 10^{-2}$, (b) $A=\pi^{2}, B=2 \pi^{2} \times 10^{-2}$, (c) $A=\pi^{2} \times 10^{-3}, B=2 \pi^{2}$, and (d) $A=\pi^{2} \times 10^{-1}, B=2 \pi^{2}$. Solid curves denote the theoretical solution $S_{t}$, the positive solution of the quintic equation (32). The locations of $R_{T 1}$ and $R_{T 2}$ are indicated by the vertical lines. The types of the dominant dynamical balance are indicated by C1, C0, G1, G0, H1, and H0. Dotted curves denote Matsuda's (1980) estimates $S_{M}$, which we discuss in Section 5.4.

Similar to the case of $S_{n}$, we introduce $R_{v B n}$ and $R_{v T n}$ as follows:

$$
\begin{aligned}
& R_{v B n} \equiv-\frac{1}{a \Omega} \sum_{j=1}^{J} v\left(\phi_{j}, z_{1}\right) \cos \phi_{j} \Delta \phi_{j}, \\
& R_{v T n} \equiv \frac{1}{a \Omega} \sum_{j=1}^{J} v\left(\phi_{j}, z_{K}\right) \cos \phi_{j} \Delta \phi_{j},
\end{aligned}
$$

where $z_{1}$ is the altitude of the lowest layer. Figure 8 shows the dependence of $R_{v B n}$ (circle) and $R_{v T n}$ (square) on $R_{T}$ for (a)-(d) with the theoretical estimates [i.e., $R_{v B t}$ and $R_{v T t}$ obtained by substituting $S_{t}$ into Eqs. (19) and (21); solid and dashed curves, respectively]. The theoretical estimation is qualitatively correct; $R_{v B n}$ is nearly equal to $R_{v T_{n}}$ when $S_{n} \ll 1$, whereas $R_{v B n}$ is much larger than $R_{v T_{n}}$ when $S_{n} \gg 1$. The estimation errors of $R_{v B}$ and $R_{v T}$ are slightly larger than those of $S$.

For the obtained numerical solutions, $\beta_{n}$ is introduced as

$$
\beta_{n} \equiv \frac{1}{\Theta_{0} \Delta_{H}} \sum_{k=1}^{K} \frac{\theta\left(\phi_{1}, z_{k}\right)-\theta\left(\phi_{J}, z_{k}\right)}{K},
$$


(a)

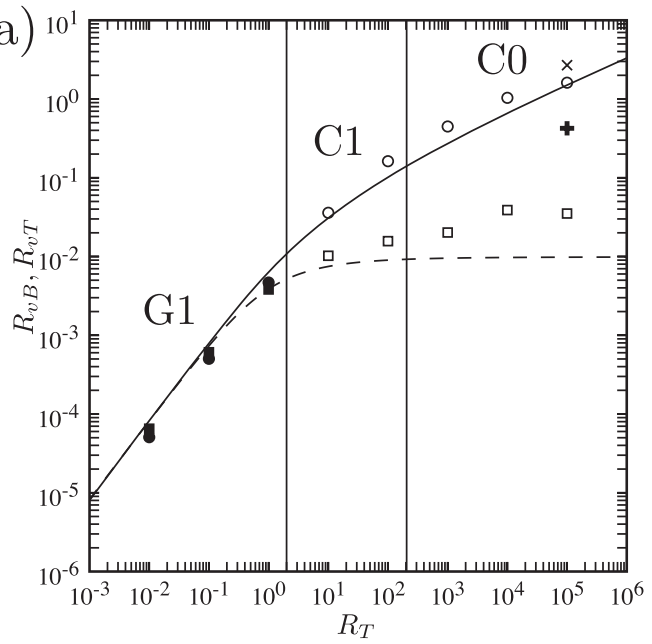

(b)

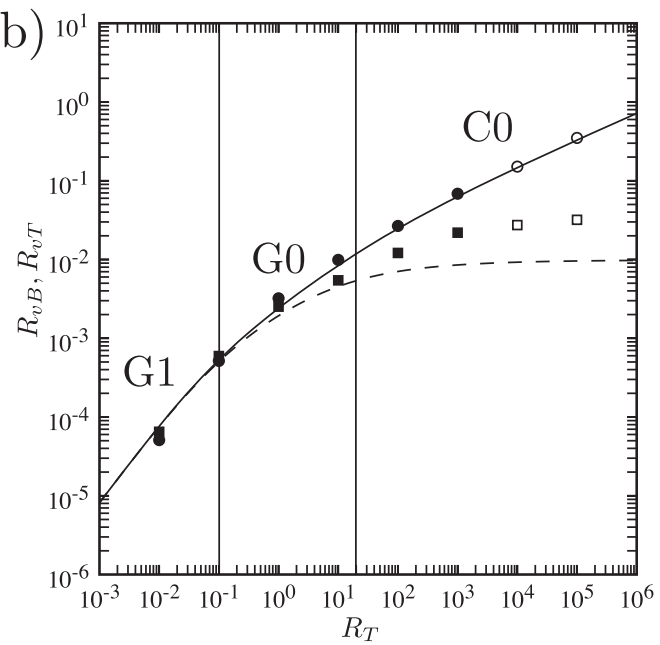

(c)

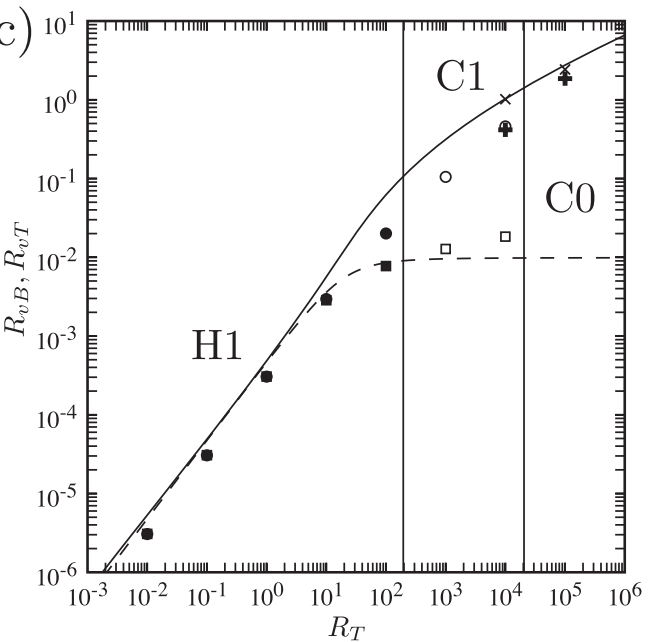

(d)

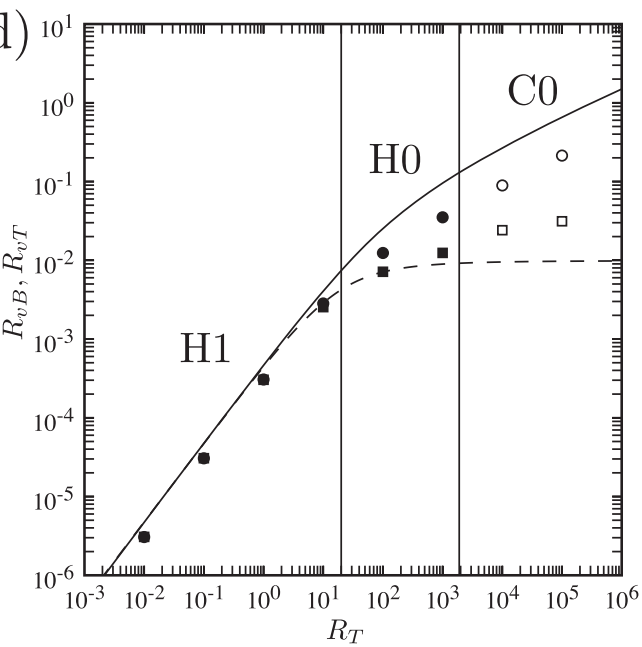

Fig. 8. Same as Fig. 7 but for $R_{v B_{n}}$ (solid circle, open circle, and cross symbol) and $R_{v T_{n}}$ (solid square, open square, and plus symbol are used instead). Solid curves denote $R_{v B t}$ and dashed curves denote $R_{v T t}$.

where $z_{k}(k=1,2,3, \ldots, K ; K=50)$ is the altitude at each layer. Figure 9 shows the dependence of $\beta_{n}$ on $R_{T}$ for (a)-(d) with the theoretical estimates $\beta_{t}$, which is obtained by substituting $S_{t}$ into Eq. (33). When the dynamical balance is $\mathrm{C} 1, \mathrm{G} 1$, or $\mathrm{H} 1, \beta_{n} \approx 1$ is in good agreement with our theoretical model, whereas when the dynamical balance is $\mathrm{C} 0, \mathrm{G} 0$, or $\mathrm{H} 0, \beta_{n}$ tends to decrease from $\beta_{n} \approx 1$ as $R_{T}$ increases.

\subsection{Dependence on $E_{V}$}

Our theoretical model predicts that $S$ and $\beta$ depend only on $A, B$, and $R_{T}$, as given by Eqs. (32) and (33), whereas $R_{v B}$ and $R_{v T}$ depend explicitly on $E_{V}$ in addition to $A, B$, and $R_{T}$, as given by Eqs. (19) and (21). To verify this prediction, another series of numerical time- integrations are performed with $A=\pi^{2} \times 10^{-1}, B=$ $2 \pi^{2}$, and $E_{V}=10^{-2}$, labeled by $\left(\mathrm{d}^{\prime}\right)$ in Table 2. The values of $A$ and $B$ are same as those of (d), whereas $E_{V}$ of $\left(d^{\prime}\right)$ is ten times larger than that of (d). Table 3 shows the ratios of $S_{n}, R_{v B n}, R_{v T_{n}}$, and $\beta_{n}$ for (d') to those for (d). The ratios of $S_{n}$ and $\beta_{n}$ are about one, and the ratios of $R_{v B n}$ and $R_{v T_{n}}$ are about ten. These results are consistent with our theoretical model estimation. The ratio of $R_{v B n}$ is smaller than ten because the larger $E_{V}$ (i.e., the larger vertical diffusion coefficient) leads to a deeper boundary layer, and hence the altitude of the maximum equatorward wind speed is raised. For example, when $E_{V}=10^{-3}$, the altitude of the maximum equatorward wind speed is $z_{1}$, where $R_{v B n}$ is calculated, whereas when $E_{V}=10^{-2}$, the altitude is $z_{2}$, which is one layer 

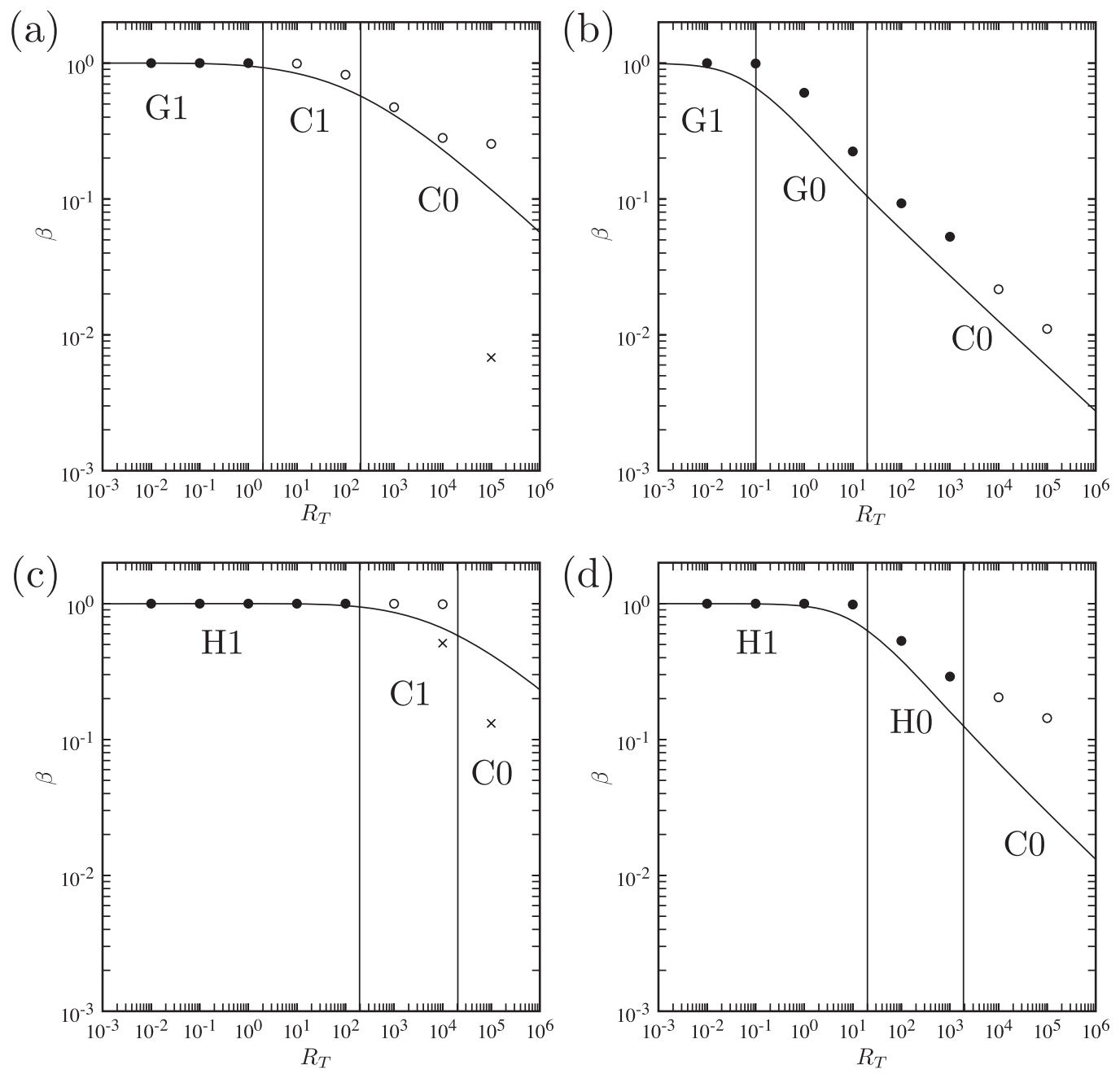

Fig. 9. Same as Fig. 7 but for $\beta_{n}$. Solid curves denote $\beta_{t}$.

Table 3. Ratios of $S_{n}, R_{v B n}, R_{v T_{n}}$, and $\beta_{n}$ for (d') to those for (d), respectively. Both $(\mathrm{d})$ and $\left(\mathrm{d}^{\prime}\right)$ have the same values of $A=\pi^{2}$ $\tau \Omega E_{V}=\pi^{2} \times 10^{-1}$ and $B=20 \pi^{2} E_{H} E_{V}=2 \pi^{2}$, whereas $E_{V}$ of $\left(\mathrm{d}^{\prime}\right)$ is ten times larger than that of $(\mathrm{d})$.

\begin{tabular}{c|cccc}
\hline & \multicolumn{4}{|c}{ Ratios of } \\
$R_{T}$ & $S_{n}$ & $R_{v B n}$ & $R_{v T n}$ & $\beta_{n}$ \\
\hline $10^{-2}$ & 0.98 & 7.36 & 9.86 & 1.00 \\
$10^{-1}$ & 0.98 & 7.32 & 9.86 & 1.00 \\
$10^{0}$ & 0.98 & 7.36 & 9.86 & 1.00 \\
$10^{1}$ & 0.98 & 7.41 & 9.89 & 1.00 \\
$10^{2}$ & 0.98 & 7.18 & 10.24 & 1.13 \\
$10^{3}$ & 0.98 & 7.27 & 9.30 & 1.14 \\
$10^{4}$ & 1.01 & 7.57 & 9.41 & 1.14 \\
$10^{5}$ & 0.97 & 7.56 & 9.40 & 0.97 \\
\hline
\end{tabular}

above $z_{1}$ for all $R_{T}$ from $10^{-2}$ to $10^{5}$.

\section{Discussion}

\subsection{Formulation of eddy diffusion}

The formulation of the eddy diffusion terms is a key assumption in this study. Both the theoretical and numerical results obtained in the previous sections depend on the form of the eddy diffusion terms, which represent the effect of nonaxisymmetric eddy mixing. The following three characteristics of the eddy diffusion terms used in this study are essential for the Gierasch mechanism to work. The horizontal diffusion 1) does not transport heat, 2) can transport $M$ (absolute angular momentum) in its up-gradient direction, and 3) is much stronger than both the vertical diffusion and the meridional advection. 
We have used the horizontal diffusion that strongly transports momentum but does not transport heat. This assumption is also used in Gierasch (1975) and is important to obtain strong superrotation. If the horizontal eddy diffusion of heat is as strong as that of momentum, the equator-to-pole temperature difference will be decreased immediately by the diffusion. Without a significant equator-to-pole temperature difference, the meridional circulation will not be driven; accordingly, the superrotation will not be generated or maintained by the Gierasch mechanism.

The horizontal diffusion term of zonal momentum used in this study is formed to homogenize angular velocity and can transport $M$ in its up-gradient direction. To show why this characteristic is essential for the Gierasch mechanism, we shortly review "Hide's (1969) theorem." If the flow is purely axisymmetric, the eddy diffusion terms representing nonaxisymmetric mixing in Eqs. (1) and (2) should be replaced by the molecular diffusion terms; i.e., both $\nu_{H}$ and $\nu_{V}$ should be replaced by the molecular diffusion coefficient $\nu$. In such a case, because $a \gg H$ for the present geometry of a shallow fluid on a sphere, vertical derivatives are dominant in the molecular diffusion terms and the terms with horizontal derivatives can be ignored. As a result, the angular momentum equation for a steady, purely axisymmetric flow becomes

$$
\frac{1}{a \cos \phi} \frac{\partial}{\partial \phi}(v M \cos \phi)+\frac{\partial}{\partial z}(w M)=\nu \frac{\partial^{2} M}{\partial z^{2}},
$$

which consists of the advection terms (LHS) and the vertical diffusion term (RHS) that only transports $M$ in its down-gradient direction. ${ }^{3}$ Hide's theorem states that $M$ cannot have a local extremum in the interior or at the stress-free boundary of the fluid of a steady, purely axisymmetric flow governed by the above equation. If there is a local extremum in the interior, inconsistency would arise in the above equation. That is, an integration of the advection terms (LHS) along a closed contour of constant $M$ around the extremum vanishes but that of the down-gradient diffusion term (RHS) never vanishes. Similarly, Hide's theorem also precludes a local extremum of $M$ at the stress-free upper boundary. Therefore, the superrotation state

\footnotetext{
${ }^{3}$ In cases that length scales of horizontal and vertical are comparable, such as in the geometry of cylindrical fluid annulus in a laboratory (Read 1986a), the horizontal part of the molecular diffusion is significant and can transport $M$ in its upgradient direction. Therefore, Hide's theorem is not applicable in such cases.
}

cannot be obtained in a steady, purely axisymmetric flow. [See Lindzen (1990, Chap. 7.2.1) and Vallis (2006, Chap. 11.2.8) for details.] The key precondition of the theorem is that the dominant diffusion term transports $M$ only in its down-gradient direction. The theorem is also applicable to a steady flow of a quasiaxisymmetric model if the direction of angular momentum transport by dominant eddy diffusion is only down-gradient. However, in this study, the horizontal eddy diffusion can transport $M$ in its upgradient direction and is dominant; therefore, Hide's theorem is not applicable and the superrotation has been obtained.

The strength of the horizontal eddy diffusion of momentum is also important. We have used the assumption that the relaxation time for horizontal diffusion is much shorter than both the turnover time of the meridional circulation and the relaxation time for vertical diffusion, as Gierasch (1975) stated. If the turnover time of the meridional circulation is not sufficiently long, the angular momentum in the upper atmosphere will be transported poleward and then downward by the meridional circulation, and the (strong) superrotation will not be obtained. The shallow-jet solutions (a) $R_{T}=10^{5}$ and (c) $R_{T} \geq 10^{4}$ obtained in Section 4.2 might correspond to this situation. If the vertical diffusion is not sufficiently weak, the angular momentum will be removed downward to the surface by the vertical diffusion.

Before concluding this subsection, we should note that there may be several ways to formulate the eddy diffusion terms that parameterize the effect of nonaxisymmetric eddy mixing. Even in 3D models, there are various ways to formulate the eddy diffusion terms that parameterize the effect of subgrid-scale eddies (Jablonowski and Williamson 2011). We have formulated the horizontal eddy diffusion term of zonal momentum (6) to homogenize angular velocity while conserving the meridional integral of $M$ from the south pole to the north pole. Form (6) is equivalent to that used in Gierasch (1975) and also equivalent to the form that mixes vorticity while conserving the meridional integral of $M$, such as Eq. (3.13) in Becker (2001) and Eq. (13.14) in Jablonowski and Williamson (2011) under the assumption of $\partial / \partial \lambda=0$, where $\lambda$ is the longitude. The conservation of the meridional integral of $M$ is the physical principle that any horizontal eddy diffusion process must satisfy, though some of the eddy diffusion forms used in GCMs violate the principle (Becker 2001; Jablonowski and Williamson 2011). It is true that the results obtained in this study depend on the form of the eddy diffusion. However, as 
long as the diffusion terms have the three characteristics discussed in this subsection, the impact of the form of the eddy diffusion on our results should be minor.

\subsection{Assumptions in the theoretical model}

To develop our theoretical model that consists of Eqs. (19), (21), (26), and (31), we have assumed a steady state $(\partial / \partial t=0)$ and the following spatial structures, each of which is written in a trigonometric function:

- the meridional integral of relative angular momentum: $M_{0}(z)=U a[1-\cos (\pi z / H)] / 2$,

- the zonal wind at the top: $u(\phi, H)=(3 U / 2) \cos \phi$,

- the meridional wind just above the surface: $v(\phi, h)=-V_{B} \sin 2 \phi$,

- the meridional wind at the top: $v(\phi, H)=V_{T} \sin 2 \phi$, and

- the vertically averaged potential temperature: $\bar{\theta}(\phi)=\Theta_{0}-\Delta \Theta\left(\sin ^{2} \phi-1 / 3\right)$.

As noted in the previous section, we have obtained steady-state solutions and statistically steady-state solutions as numerical solutions of governing equations (1)-(11). The statistically steady-state solutions do not exactly satisfy the steady-state assumption, but the oscillation amplitudes for $S_{n}$ in the time-dependent solutions are less than $3 \%$. This means that the oscillation has little effect on superrotation strength.

To evaluate how close the numerical solutions are to the assumed spatial structure of each function, we calculate the measures for closeness defined as

$$
\begin{aligned}
& E\left[M_{0}(z)\right] \equiv \sqrt{\sum_{k=1}^{K} \frac{\left\{\hat{M}_{0}\left(z_{k}\right)-\left[1-\cos \left(\pi z_{k} / H\right)\right] / 2\right\}^{2}}{K}}, \\
& E[u(\phi, H)] \equiv \sqrt{\sum_{j=1}^{J} \frac{\left[\hat{u}\left(\phi_{j}, z_{k}\right)-\cos \phi_{j}\right]^{2}}{J}}, \\
& E[v(\phi, h)] \equiv \sqrt{\sum_{j=1}^{J} \frac{\left[\hat{v}\left(\phi_{j}, z_{1}\right)+\sin 2 \phi_{j}\right]^{2}}{J}}, \\
& E[v(\phi, H)] \equiv \sqrt{\sum_{j=1}^{J} \frac{\left[\hat{v}\left(\phi_{j}, z_{K}\right)-\sin 2 \phi_{j}\right]^{2}}{J}}, \\
& E[\bar{\theta}(\phi)] \equiv \sqrt{\sum_{j=1}^{J} \frac{\left\{\left[\bar{\theta}\left(\phi_{1}\right)-\bar{\theta}\left(\phi_{j}\right)\right] /\left[\bar{\theta}\left(\phi_{1}\right)-\bar{\theta}\left(\phi_{J}\right)\right]-\sin ^{2} \phi_{j}\right\}^{2}}{J}},
\end{aligned}
$$

for the numerical solutions. Here, hat $(\hat{)})$ means "normalized by its maximum among the absolute values of the summed values." Table 4 shows the maximum and the median for each measure among the deep-jet solutions. Aside from $E[v(\phi, H)]$, the maxima are less than 0.19 and the medians are less than 0.07 . These mean that the spatial structures of $M_{0}(z), u(\phi, H)$, $v(\phi, h)$, and $\bar{\theta}(\phi)$ in the numerical solutions are close to
Table 4. Maximum and median (among the deep-jet solutions) of measures for closeness to the assumed functions. See text for each definition.

\begin{tabular}{l|cc}
\hline & Maximum & Median \\
\hline$E\left[M_{0}(z)\right]$ & 0.149 & 0.048 \\
$E[u(\phi, H)]$ & 0.003 & 0.001 \\
$E[v(\phi, h)]$ & 0.187 & 0.065 \\
$E[v(\phi, H)]$ & 0.689 & 0.211 \\
$E[\bar{\theta}(\phi)]$ & 0.049 & 0.009 \\
\hline
\end{tabular}

the assumed functions. The high $E[v(\phi, H)]$ is marked when the distorted meridional cell appears, such as (a) $R_{T} \geq 10^{3}$. We present a possible reason for the appearance of the distorted meridional cell in the next subsection.

\subsection{Distortion of the meridional cell and static instability}

As shown in Fig. 4 and described in Section 4.2, for deep-jet solutions, the meridional cell symmetric with respect to the mid-level is gradually distorted and becomes $\Delta$-shaped as $R_{T}$ increases. We speculate that the $\Delta$-shaped meridional cell can be a combined result of two processes described below.

The first is an asymmetrization process with respect to the mid-level. Our theoretical model states that the equatorward flow just above the bottom surface $\left(R_{v B}\right)$ is faster than the poleward flow at the top $\left(R_{v T}\right)$, as given by Eqs. (19) and (21). In Fig. 8, the difference between $R_{v B}$ and $R_{v T}$ becomes apparent when $S \gtrsim 1$ or

$$
R_{v B} \gtrsim \pi^{2} E_{V},
$$

from Eq. (19). In this case, because of the continuity equation (5), the upper-poleward flow must be thicker than the lower-equatorward flow, as illustrated in Fig. 10 (left). A typical example in the numerical solutions is (a) $R_{T}=10^{\circ}$ in Fig. 4.

The second is an inclination process. Let us consider the potential temperature difference between the upper part and the lower part of the atmosphere. Remember that the Newtonian heating and cooling process relaxes the atmosphere to the radiative-convective equilibrium state $\theta_{e}$ given by Eq. (8); that is, the atmosphere is heated in low-latitudes and cooled in high-latitudes for $0<\beta<1$. Therefore, the upper flow from the equator to the pole must be warmer than the lower flow from the pole to the equator. This vertical temperature difference becomes apparent when the turnover time of the meridional circulation is shorter than the time constant for Newtonian heating and cooling, namely, 

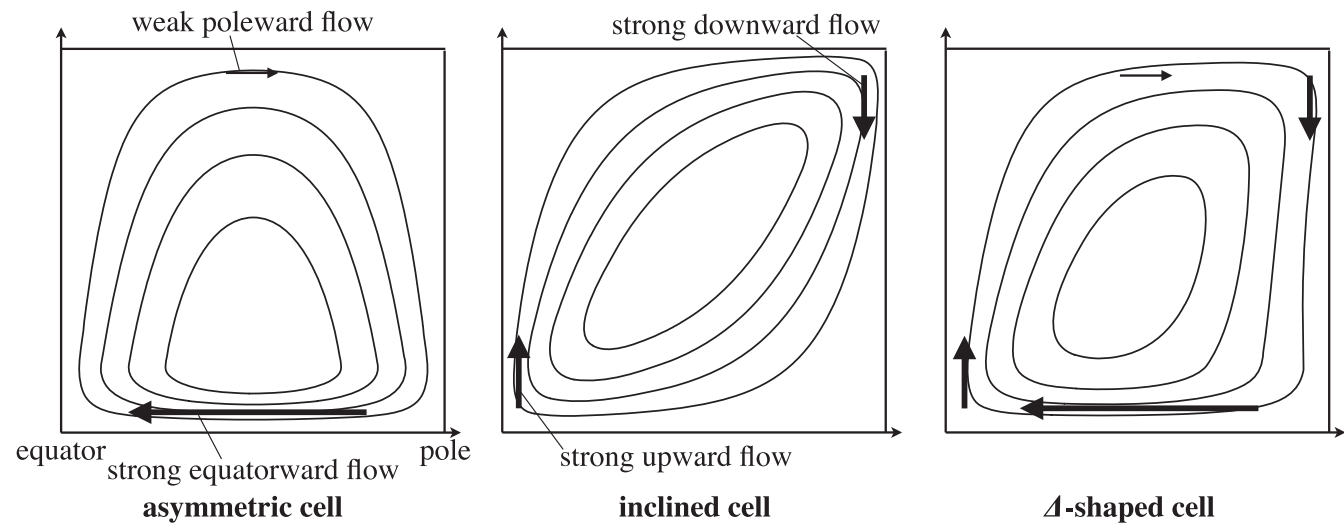

Fig. 10. Schematic illustrations of an asymmetric cell (left), an inclined cell (middle), and a $\Delta$-shaped cell (right). The $\Delta$ shaped cell is the mean of the asymmetric cell and the inclined cell.

$$
R_{v B}>(\tau \Omega)^{-1}
$$

in nondimensional parameters. Because the radiativeconvective equilibrium state is specified in a statically neutral state $\left(\partial \theta_{e} / \partial z=0\right)$, the difference $\left|\theta-\theta_{e}\right|$ in the lower atmosphere at the equator and that in the upper atmosphere at the pole become larger than that in the rest of the atmosphere. In the lower atmosphere at the equator, a strong upward flow is required by the thermodynamic equation (4) under the steady-state assumption, whereas in the upper atmosphere at the pole, a strong downward flow is required. As a consequence, the meridional cell inclines as illustrated in Fig. 10 (middle). A typical example is (b) $R_{T}=10^{-1}$ in Fig. 4. This kind of inclined meridional cell is also shown by Sugata and Yoden (1992) in a numerical solution of a rotating annulus experiment with radial differential heating.

Now, we speculate that the superposition of the asymmetric cell and the inclined cell gives the $\Delta$ shaped cell as illustrated in Fig. 10 (right), which is the mean of the streamfunction of the asymmetric cell (Fig. 10 left) and that of the inclined cell (Fig. 10 middle).

A linear superposition is not always guaranteed because the above two processes include thermal advection, which is a nonlinear process. However, the spatial structure of the meridional circulation in the numerically obtained deep-jet solutions (Fig. 4) qualitatively agrees with the picture described above. For example, in the series (a), in which the parameter $\pi^{2}$ $E_{V}=\pi^{2} \times 10^{-3}$ is smaller than $(\tau \Omega)^{-1}=10^{-1}$, a meridional cell asymmetric with respect to the mid-level appears first when $R_{T}=10^{\circ}$, and then a $\Delta$-shaped cell appears when $R_{T} \geq 10^{1}$. On the other hand, in the series (b), in which $\pi^{2} E_{V}=\pi^{2} \times 10^{-3}$ is larger than $(\tau \Omega)^{-1}=10^{-3}$, an inclined cell appears first when $R_{T}=$ $10^{-1}$, and then a $\Delta$-shaped cell appears when $R_{T} \geq$ $10^{\circ}$.

In addition to the distortion process described above, we speculate that the $\Delta$-shaped meridional cell leads the atmosphere in low- and mid-latitudes to a statically unstable state. In the $\Delta$-shaped cell, warm air flows from the equator to mid-latitudes at a low altitude, as illustrated in Fig. 10 (right). Such a warm air at a low altitude can cause static instability. As an example, Fig. 11 shows static stability $(\partial \theta / \partial z)$ and the meridional streamfunction for (a) $R_{T}=10^{1}$ at $t=20000$ days. Buoyant convection occurs in the region where $\partial \theta / \partial z$ is negative (shaded region). Thus, the $\Delta$-shaped meridional cell can prevent the solution from being a steady state. We should note that the static instability discussed above is a possible explanation, whereas another explanation might be possible using, for example, the concept of viscous overturning described in McIntyre (1970a, b).

\subsection{Comparison with Matsuda (1980)}

There are the following four major differences between the theoretical model introduced in Section 3 and that in Matsuda (1980) (hereafter referred to as M80), in which the parameter dependence of superrotation strength was theoretically explored using a highly truncated spectral model. ${ }^{4}$

1. The equator-to-pole temperature difference is an internal variable in this study, while it was fixed as an external condition in M80.

2. The number of modes of the zonal wind at the top 


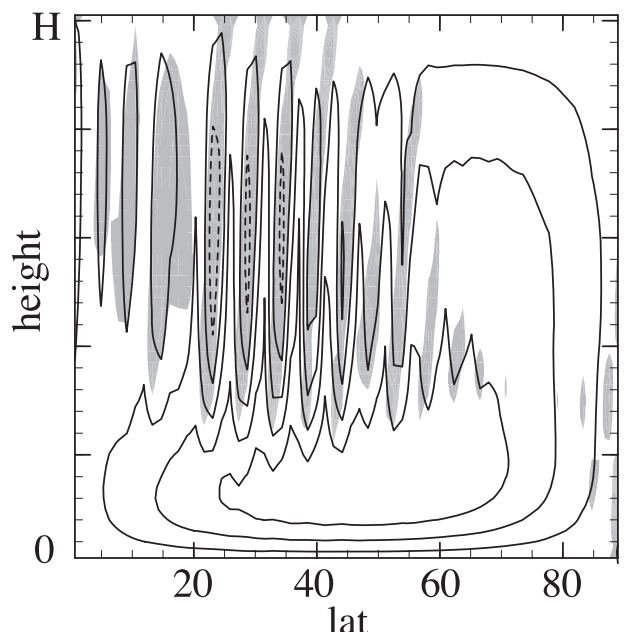

Fig. 11. Nondimensional meridional streamfunction $\Psi(\phi, z) /(a H \Omega)$ (contour) for $A=\pi^{2} \times 10^{-2}, B=2 \pi^{2} \times 10^{-2}$, and $R_{T}=10^{1}$ at $t=20000$ days. Here, $v=-\partial \Psi / \partial z$ and $w=(a \cos \phi)^{-1} \partial(\Psi \cos \phi) / \partial \phi$. Contour interval is $4.28 \times 10^{-3}$. Dashed contour indicates a negative value. The shaded area indicates a statically unstable $(\partial \theta / \partial z<0)$ region.

is one (solid-body rotation mode) in this study, while it is two (solid-body and differential rotation modes) in M80.

3. The horizontal diffusion for the meridional wind is included in this study, while it was not included in M80.

4. The scales for the meridional winds just above the surface $\left(V_{B}\right)$ and at the top $\left(V_{T}\right)$ are treated individually in this study, while $V_{B}=V_{T}$ were assumed in M80.

Matsuda (1980) presented an equation for the superrotation strength for a finite horizontal diffusion case (Eq. 4.17 in M80); let us denote the solution of his equation by $S_{M}$. Dotted curves on Fig. 7 show the dependence of $S_{M}$ on $R_{T}$. Note that negative values of $S_{M}$ are not shown, which correspond to the other stable solution of multiple equilibrium states in M80, and $S_{M}$ has the maximum value at certain $R_{T}$, which corresponds to the upper limit due to the differential

\footnotetext{
${ }^{4}$ In M80, three different situations with respect to horizontal eddy diffusion were considered: one with infinite diffusion for the zonal wind, one with finite diffusion for the zonal wind, and one with finite diffusion acting equally on the zonal and meridional winds and the potential temperature. We compare our model with the second situation because his major results were obtained from the second one.
}

rotation mode in Matsuda's model with finite horizontal diffusion. When the dynamical balance is C1 or G1, $S_{M}$ as well as $S_{t}$ agrees well with $S_{n}$; that is, Matsuda's estimation is also good for these cases. In contrast, when the dynamical balance is G0, H1, or H0, $S_{M}$ does not agree with $S_{n}$, whereas $S_{t}$ agrees well with $S_{n}$. These results are understandable for the following reasons. Because the effect of thermal advection was ignored and the equator-to-pole temperature difference was fixed to a constant value in $\mathrm{M} 80, S_{M}$ must differ from $S_{n}$ when the thermal advection is dominant $(\mathrm{G} 0$, $\mathrm{H} 0$ ). Because the horizontal diffusion term was included only in the zonal momentum equation in M80, $S_{M}$ differs from $S_{n}$ when the dominant dynamical balance is the horizontal diffusion balance $(\mathrm{H} 1, \mathrm{H} 0)$, which is the balance between the pressure gradient term and the horizontal diffusion term in the meridional momentum equation. Our numerical results show that superrotation strength can exceed the upper limit predicted in M80 when $R_{T}$ is large enough. The difference in the treatment of the scales of meridional winds is not significant for the determination of superrotation strength. However, two scales $V_{B}$ and $V_{T}$ have enabled us to discuss the distortion of the meridional cell in the previous subsection.

\subsection{Note on applying the theoretical model to real atmospheres}

The superrotation strength estimated by Eq. (32) is $S \approx 60-140$ for Venus-like parameter values roughly assumed as $A=10^{-3}-10^{-1}, B=10^{-1}$, and $R_{T}=10^{4}$. For this estimation, the following nondimensional values are used: $\tau \Omega=10^{-1}-10^{1}$ (Sánchez-Lavega 2010; Table 4.3), $E_{H}=10^{\circ}$, and $E_{V}=10^{-3}$. Here, the variation of $\tau$ with respect to height is considered, and the values of $R_{T}, E_{H}$, and $E_{V}$ are just guesses. Similarly, assuming Titan-like parameter values as $A=10^{1}-10^{2}, B=10^{-1}$, and $R_{T}=10^{2}$ with $\tau \Omega=10^{3}-10^{4}, E_{H}=10^{\circ}$, and $E_{V}=$ $10^{-3}$, we obtain $S \approx 0.9-2.5$. These estimates of the superrotaion for the Venus and Titan atmospheres are not quite different from the observed superrotation strength. However, we should note that our theoretical model has been constructed under many assumptions and approximations. (Of course, they have been necessary for the construction of the theoretical model.) For example, we have used the Boussinesq approximation, although the density varies by a few orders of magnitude in the atmospheres of Venus and Titan. To evaluate the impact of the approximation on superrotation strength and the dominant dynamical balance, which we have investigated in this study, careful comparison of numerical solutions between 
models with and without the Boussinesq approximation is needed.

For rapidly rotating planets $\left(R_{T}<1\right)$, a further consideration is needed. This is because we have assumed strong horizontal eddy diffusion representing zonally averaged equatorward transport of angular momentum and a single Hadley cell extending to the pole. These assumptions are not valid at least for the atmosphere of the present Earth. ${ }^{5}$ However, a tropical superrotating state found in a terrestrial two-level model by Suarez and Duffy (1992) and Saravanan (1993) might correspond to the solutions for $R_{T}<1$ in this study. They showed the tropical superrotating state when large zonally asymmetric tropical heating exists, which causes zonally averaged equatorward transport of angular momentum. If such angular momentum transport plays the role of the strong horizontal eddy diffusion, the tropical superrotating state corresponds to the solutions for $R_{T}<1$ in this study.

\section{Summary}

We have theoretically estimated the strength of the planetary atmospheric superrotation that is maintained by the Gierasch (1975) mechanism from the quasiaxisymmetric primitive equations for a dry Boussinesq fluid on a hemisphere with Newtonian heating and cooling and with strong horizontal eddy momentum diffusion parameterizing horizontal mixing by nonaxisymmetric eddies [Eqs. (1)-(11)]. From the governing PDEs, four relationship equations (19), (21), (26), and (31) have been obtained by assuming a steady state and the following scales and functions about the general circulation of planetary atmospheres: the meridional integral of relative angular momentum $M_{0}(z)=$ $U a[1-\cos (\pi z / H)] / 2$, the zonal wind at the top $u(\phi, H)=(3 U / 2) \cos \phi$, the meridional winds just above the surface $v(\phi, h)=-V_{B} \sin 2 \phi$ and at the top $v(\phi, H)=$ $V_{T} \sin 2 \phi$, and the vertically averaged potential temperature $\bar{\theta}(\phi)=\Theta_{0}-\Delta \Theta\left(\sin ^{2} \phi-1 / 3\right)$. The four relationship equations constitute a set of simultaneous algebraic equations having four unknown variables that characterize the general circulation of planetary atmospheres: the superrotation strength $S=U /(a \Omega)$, the Rossby number scaled with the meridional wind just above the surface $R_{v B}=V_{B} /(a \Omega)$, the Rossby number

\footnotetext{
${ }^{5}$ For the terrestrial Hadley circulation, Held and Hou (1980) presented a theoretical model using a nearly inviscid axisymmetric system without horizontal diffusion, and Yamamoto et al. (2009) numerically explored the the connection in a parameter space $\left(R_{T}-E_{H}\right.$ space $)$ between the Gierasch mechanism and Held and Hou's model.
}

scaled with the meridional wind at the top $R_{v T}=$ $V_{T} /(a \Omega)$, and the ratio of the equator-to-pole difference of potential temperature to that in a prescribed radiative-convective equilibrium state $\beta=\Delta \Theta /\left(\Theta_{0} \Delta_{H}\right)$.

We have obtained the quintic equation (32) for $S$ by eliminating the other unknown variables. The positive solution of the quintic equation estimates superrotation strength, which depends only on the external thermal Rossby number $R_{T}=g H \Delta_{H} /\left(a^{2} \Omega^{2}\right)$ and the two external nondimensional parameters $A=\pi^{2} \tau \Omega E_{V}$ and $B=20 \pi^{2}$ $E_{H} E_{V}$. By approximating the quintic equation (32) to the cubic equation (35), we have obtained an analytical form of the estimate for the four cases (a)-(d) given in Table 1 and shown in Fig. 2. We have also revealed the parameter dependence of the dominant dynamical balance, which is a cyclostrophic $[\mathrm{C}]$, geostrophic $[\mathrm{G}]$, or horizontal diffusion $[\mathrm{H}]$ balance. In each balance, the equator-to-pole temperature difference is either nearly equal to that in the radiative-convective equilibrium state $\beta \approx 1[1]$ or is significantly reduced by thermal advection $\beta \ll 1[0]$. The parameter dependence of the balance is shown in Table 1 and Fig. 2.

To test the validity of the above estimation, we have numerically obtained steady-state or statistically steady-state solutions of the primitive equations (1)(11) for $R_{T}=10^{n}(n=-2,-1,0, \ldots, 5)$ with four combinations of values of $A$ and $B$ listed in Table 2 (a)(d). From the numerical solutions (shown in Figs. 3-6), the superrotation strength $S$ has been calculated and compared with the theoretical estimates, as shown in Fig. 7. Within the parameter range, the theoretical estimates agree well with the numerically obtained superrotation strength. The relative error of the estimates is less than $50 \%$, even though we have calculated the parameter range covering many orders of magnitude. Similar to the superrotation strength, the numerically obtained $R_{v B}, R_{v T}$, and $\beta$ are well estimated by the theoretical model, as shown in Figs. 8 and 9.

The numerical solutions that did not achieve a steady state have a statically unstable region in the atmosphere, as shown in Fig. 11, for example. Such an unstable region is developed by the lower-poleward branch in the $\Delta$-shaped meridional cell (Fig. 10 right), which can be explained by the superposition of the asymmetric cell (Fig. 10 left) and the inclined cell (Fig. 10 middle). The asymmetric cell results from the speed gap between the upper-poleward flow (slow and thick) and the lower-equatorward flow (fast and thin), whereas the inclined cell results from strong Newtonian cooling in the upper atmosphere at the pole and strong Newtonian heating in the lower atmosphere at 
the equator.

Multiple equilibrium solutions that we have obtained support the possibility of multiple stable equilibrium states in the Venus atmosphere shown by Matsuda $(1980,1982)$ by using a two-layer highly truncated system and by Kido and Wakata (2008, 2009) by using a Venus-like 3D GCM. The degrees of freedom of our quasi-axisymmetric system is much greater than that of Matsuda's system and is much less than that of the Venus-like 3D GCM. Therefore, our finding supports the possibility of multiple equilibrium states in planetary atmospheres.

Our theoretical model gives $S \approx 60-140$ for Venuslike parameter values and $S \approx 0.9-2.5$ for Titan-like parameter values. These estimates of the superrotaion strength are not quite different from the observed values. However, we should note that our theoretical model has been constructed under many assumptions and approximations, which may affect the estimates. To evaluate their impacts, a comparison study using models with various levels of idealization is needed as our future work.

\section{Acknowledgements}

We thank Professors Yoshihisa Matsuda, Takeshi Horinouchi, and Keiichi Ishioka, Doctor Shigenori Otsuka, and anonymous reviewers for useful comments. The figures were produced by the GFDDENNOU Library and mjograph. This work was supported by the JSPS KAKENHI Grant-in-Aid for JSPS Fellows 21·393.

\section{Appendix: Demonstration that Eq. (32) has only one positive solution}

The LHS of Eq. (32) can be expanded as follows ( $S$ is replaced by $x$ for readability):

$$
\begin{aligned}
& \text { LHS of Eq. (32) } \\
& =\left[x^{2}+2 x+B x\left(\frac{2+x}{1+x}\right)\right]\left[\frac{A}{2} x\left(\frac{2+x}{1+x}\right)+1\right] \\
& =\frac{1}{(1+x)^{2}}\left[x^{3}+(B+3) x^{2}+2(1+B) x\right]\left[\frac{A}{2} x^{2}+(A+1) x+1\right] \\
& =\frac{1}{(1+x)^{2}}\left[C_{1} x^{5}+C_{2} x^{4}+C_{3} x^{3}+\left(C_{4}+B+1\right) x^{2}+2(B+1) x\right],
\end{aligned}
$$

where $C_{1}, C_{2}, C_{3}$, and $C_{4}$ are positive constants. If we define $f(x)$ as

$$
\begin{aligned}
f(x) \equiv & {\left[C_{1} x^{5}+C_{2} x^{4}+C_{3} x^{3}+\left(C_{4}+B+1\right) x^{2}+2(B+1) x\right] } \\
& -2 R_{T}(1+x)^{2}
\end{aligned}
$$

the solution of $f(x)=0$ is the solution of Eq. (32). The differentiation of $f(x)$ can be written as

$$
\begin{aligned}
\frac{d f}{d x}= & 5 C_{1} x^{4}+4 C_{2} x^{3}+3 C_{3} x^{2}+2\left(C_{4}+B+1-2 R_{T}\right) x \\
& +2\left(B+1-2 R_{T}\right) .
\end{aligned}
$$

In the case that $\left(B+1-2 R_{T}\right) \geq 0, f(x)$ increases monotonically for $x>0$; otherwise, $f(x)$ has exactly one local extremum (which is a negative local minimum) at $x=x_{0}$ for $x>0$ and increases monotonically for $x>x_{0}$ because $d f /\left.d x\right|_{x=0}$ is negative and the coefficients of $x^{4}, x^{3}$, and $x^{2}$ in $d f / d x$ are positive (i.e., $d f / d x>0$ for $x>x_{0}$, where $d f /\left.d x\right|_{x=x_{0}}=0$ ). In addition, $f(0)=-2 R_{T}<0$ and $f(x) \rightarrow \infty$ for $x \rightarrow \infty$; hence there exists exactly one positive $x_{S}$ that satisfies $f\left(x_{S}\right)=$ 0 .

\section{References}

Becker, E., 2001: Symmetric stress tensor formulation of horizontal momentum diffusion in global models of atmospheric circulation. J. Atmos. Sci., 58, 269-282.

Bird, M. K., M. Allison, S. W. Asmar, D. H. Atkinson, I. M. Avruch, R. Dutta-Roy, Y. Dzierma, P. Edenhofer, W. M. Folkner, L. I. Gurvits, D. V. Johnston, D. Plettemeier, S. V. Pogrebenko, R. A. Preston, and G. L. Tyler, 2005: The vertical profile of winds on Titan. Nature, 438, 800-802.

Counselman III, C. C., S. A. Gourevitch, R. W. King, G. B. Loriot, and E. S. Ginsberg, 1980: Zonal and meridional circulation of the lower atmosphere of Venus determined by radio interferometry. J. Geophys. Res., 85, 8026-8030.

Del Genio, A. D., and W. Zhou, 1996: Simulations of superrotation on slowly rotating planets: Sensitivity to rotation and initial condition. Icarus, 120, 332-343.

Fels, S. B., and R. S. Lindzen, 1974: The interaction of thermally excited gravity waves with mean flows. Geophys. Fluid Dyn., 6, 149-191.

Gierasch, P. J., 1975: Meridional circulation and maintenance of the Venus atmospheric rotation. J. Atmos. Sci., 32, 1038-1044.

Gierasch, P. J., R. M. Goody, R. E. Young, D. Crisp, C. Edwards, R. Kahn, D. McCleese, D. Rider, A. Del Genio, R. Greeley, A. Hou, C. B. Leovy, and M. Newman, 1997: The general circulation of the Venus atmosphere: An assessment. Venus II: Geology, Geophysics, Atmosphere, and Solar Wind Environment, Bougher, S. W., D. M. Hunten, and R. J. Phillips (eds.), University of Arizona Press, 459-500.

Held, I. M., 2005: The gap between simulation and understanding in climate modeling. Bull. Amer. Meteor. Soc., 86, 1609-1614.

Held, I. M., and A. Y. Hou, 1980: Nonlinear axially symmetric circulations in a nearly inviscid atmosphere. J. Atmos. Sci., 37, 515-533.

Hide, R., 1969: Dynamics of the atmospheres of the major planets with an appendix on the viscous boundary layer 
at the rigid bounding surface of an electricallyconducting rotating fluid in the presence of a magnetic field. J. Atmos. Sci., 26, 841-853.

Hollingsworth, J. L., R. E. Young, G. Schubert, C. Covey, and A. S. Grossman, 2007: A simple-physics global circulation model for Venus: Sensitivity assessments of atmospheric superrotation. Geophys. Res. Lett., 34, L05202.

Hoskins, B. J., 1983: Dynamical processes in the atmosphere and the use of models. Quart. J. Roy. Meteor. Soc., 109, $1-21$.

Hourdin, F., O. Talagrand, R. Sadourny, R. Courtin, D. Gautier, and C. P. McKay, 1995: Numerical simulation of the general circulation of the atmosphere of Titan. Icarus, 117, 358-374.

Iga, S., and Y. Matsuda, 1999: A mechanism of the superrotation in the Venus atmosphere: Meridional circulation and barotropic instability. Theor. Appl. Mech. Jpn., 48, 379-383.

Iga, S., and Y. Matsuda, 2005: Shear instability in a shallow water model with implications for the Venus atmosphere. J. Atmos. Sci., 62, 2514-2527.

Jablonowski, C., and D. L. Williamson, 2011: The pros and cons of diffusion, filters and fixers in atmospheric general circulation models. Numerical Techniques for Global Atmospheric Models. Lauritzen, P., C. Jablonowski, M. Taylor, and R. Nair (eds.), Springer, 381493.

Kido, A., and Y. Wakata, 2008: Multiple equilibrium states appearing in a Venus-like atmospheric general circulation model. J. Meteor. Soc. Japan, 86, 969-979.

Kido, A., and Y. Wakata, 2009: Multiple equilibrium states appearing in a Venus-like atmospheric general circulation model with three-dimensional solar heating. SOLA, 5, 85-88.

Lebonnois, S., F. Hourdin, V. Eymet, A. Crespin, R. Fournier, and F. Forget, 2010: Superrotation of Venus' atmosphere analyzed with a full general circulation model. $J$. Geophys. Res., 115, E06006.

Lee, C., S. R. Lewis, and P. L. Read, 2007: Superrotation in a Venus general circulation model. J. Geophys. Res., 112, E04S11.

Lindzen, R. S., 1990: Dynamics in Atmospheric Physics. Cambridge University Press, 310 pp.

Luz, D., and F. Hourdin, 2003: Latitudinal transport by barotropic waves in Titan's stratosphere. I. General properties from a horizontal shallow-water model. Icarus, 166, 328-342.

Matsuda, Y., 1980: Dynamics of the four-day circulation in the Venus atmosphere. J. Meteor. Soc. Japan, 58, 443-470.

Matsuda, Y., 1982: A further study of dynamics of the fourday circulation in the Venus atmosphere. J. Meteor. Soc. Japan, 60, 245-254.

McIntyre, M. E., 1970a: Diffusive destabilization of the baroclinic circular vortex. Geophy. Fluid Dyn., 1, 19-
57.

McIntyre, M. E., 1970b: Role of diffusive overturning in nonlinear axisymmetric convection in a differentially heated rotating annulus. Geophys. Fluid Dyn., 1, 58-89.

Nakamura, M., T. Imamura, N. Ishii, T. Abe, T. Satoh, M. Suzuki, M. Ueno, A. Yamazaki, N. Iwagami, S. Watanabe, M. Taguchi, T. Fukuhara, Y. Takahashi, M. Yamada, N. Hoshino, S. Ohtsuki, K. Uemizu, G. L. Hashimoto, M. Takagi, Y. Matsuda, K. Ogohara, N. Sato, Y. Kasaba, T. Kouyama, N. Hirata, Y. Yamamoto, N. Okada, T. Horinouchi, M. Yamamoto, and Y. Hayashi, 2011: Overview of Venus orbiter, Akatsuki. Earth Planets Space, 63, 443-457.

Nakamura, M., T. Imamura, M. Ueno, N. Iwagami, T. Satoh, S. Watanabe, M. Taguchi, Y. Takahashi, M. Suzuki, T. Abe, G. L. Hashimoto, T. Sakanoi, S. Okano, Y. Kasaba, J. Yoshida, M. Yamada, N. Ishii, T. Yamada, K. Uemizu, T. Fukuhara, and K. Oyama, 2007: Planet-C: Venus Climate Orbiter mission of Japan. Planet. Space Sci., 55, 1831-1842.

Read, P. L., 1986a: Super-rotation and diffusion of axial angular-momentum: I. 'Speed limits' for axisymmetrical flow in a rotating cylindrical fluid annulus. Quart. J. Roy. Meteor. Soc., 112, 231-252.

Read, P. L., 1986b: Super-rotation and diffusion of axial angular-momentum: II. A review of quasi-axisymmetrical models of planetary atmospheres. Quart. J. Roy. Meteor. Soc., 112, 253-272.

Rossow, W. B., and G. P. Williams, 1979: Large-scale motion in the Venus stratosphere. J. Atmos. Sci., 36, 377-389.

Sánchez-Lavega, A., 2010: An Introduction to Planetary Atmospheres. Taylor and Francis, 629 pp.

Saravanan, R., 1993: Equatorial superrotation and maintenance of the general circulation in two-level models. $J$. Atmos. Sci., 50, 1211-1227.

Suarez, M. J., and D. G. Duffy, 1992: Terrestrial superrotation: A bifurcation of the general circulation. J. Atmos. Sci., 49, 1541-1554.

Sugata, S., and S. Yoden, 1992: Steady axi-symmetrical flow due to differential heating in a rotating annulus and its dependence on experimental parameters. J. Meteor. Soc. Japan, 70, 1005-1017.

Vallis, G. K., 2006: Atmospheric and Oceanic Fluid Dynamics: Fundamentals and Large-scale Circulation. Cambridge University Press, 745 pp.

Yamamoto, H., K. Ishioka, and S. Yoden, 2009: Axisymmetric steady solutions in an idealized model of atmospheric general circulations: Hadley circulation and superrotation. Theor. Appl. Mech. Jpn., 57, 147-158.

Yamamoto, M., and M. Takahashi, 2003: The fully developed superrotation simulated by a general circulation model of a Venus-like atmosphere. J. Atmos. Sci., 60, 561-574.

Young, R. E., and J. B. Pollack, 1977: A three-dimensional model of dynamical processes in the Venus atmosphere. J. Atmos. Sci., 34, 1315-1351. 